\title{
Reducibility, Lyapunov Exponent, Pure Point Spectra Property for Quasi-periodic Wave Operator
}

\author{
Jing Li
}

Abstract. In the present paper, it is shown that the linear wave equation subject to Dirichlet boundary condition

$$
u_{t t}-u_{x x}+\varepsilon V(\omega t, x) u=0, \quad u(t,-\pi)=u(t, \pi)=0
$$

can be changed by a symplectic transformation into

$$
v_{t t}-v_{x x}+\varepsilon M_{\xi} v=0, \quad v(t,-\pi)=v(t, \pi)=0,
$$

where $V$ is finitely smooth and time-quasi-periodic potential with frequency $\omega \in \mathbb{R}^{n}$ in some Cantor set of positive Lebeague measure and where $M_{\xi}$ is a Fourier multiplier. Moreover, it is proved that the corresponding wave operator $\partial_{t}^{2}-\partial_{x}^{2}+\varepsilon V(\omega t, x)$ possesses the property of pure point spectra and zero Lyapunov exponent.

\section{Introduction}

If a self-adjoint differential operator with time-quasi-periodic coefficients can be reduced to one with constant coefficients, the spectrum property and Lyapunov exponent of the operator can be easily obtained. To this end, there are many literatures dealing with Schrödinger operator with time-quasi-periodic potential of the form

$$
\mathbf{i} \dot{u}=\left(H_{0}+\varepsilon W(\omega t, x,-\mathbf{i} \nabla)\right) u, \quad x \in \mathbb{R}^{d} \text { or } x \in \mathbb{T}^{d}=\mathbb{R}^{d} / 2 \pi \mathbb{Z}^{d},
$$

where $H_{0}=-\Delta+V(x)$ or an abstract self-adjoint (unbounded) operator and the perturbation $W$ is quasiperiodic in time $t$ and it may or may not depend on $x$ or/and $\nabla$. When $x \in \mathbb{R}^{d}$, there are many interesting and important results. See $[2,4,10,12,30$, and the references therein. When $x \in \mathbb{T}^{d}$ with any integer $d \geq 1$, it is in 13 , proved that

$$
\dot{u}=-\mathbf{i}\left(\Delta u-\varepsilon W\left(\phi_{0}+\omega t, x ; \omega\right) u\right), \quad x \in \mathbb{T}^{d}
$$

is reduced to an autonomous equation for most values of the frequency vector $\omega$, where $W$ is analytic in $(t, x)$ and quasiperiodic in time $t$ with frequency vector $\omega$. The reduction is

Received December 11, 2018; Accepted May 12, 2019.

Communicated by Cheng-Hsiung Hsu.

2010 Mathematics Subject Classification. 37K55, 35P05, 81Q15.

Key words and phrases. reducibility, quasi-periodic wave operator, KAM theory, finitely smooth, Lyapunov exponent, pure-point spectrum. 
made by means of Töplitz-Lipschitz property of operator and very hard KAM technique. The basic difficulty is that the frequencies of the unperturbed operator $-\Delta$, denoted by $\lambda_{k}(k \in \mathbb{Z})$, have multiplicity

$$
\lambda_{k}^{\sharp} \simeq|k|^{d-1} \rightarrow \infty \quad \text { as }|k| \rightarrow \infty \text { if } d>1
$$

Fortunately, the frequencies have a good separation property and

$$
\left|\lambda_{k}-\lambda_{k^{\prime}}\right| \geq 1 \quad \text { when } \lambda_{k} \neq \lambda_{k^{\prime}}
$$

When the reducibility for a linear wave equation

$$
u_{t t}=\left(-\Delta+\varepsilon V\left(\phi_{0}+\omega t, x ; \omega\right)\right) u, \quad x \in \mathbb{T}^{d},
$$

the unperturbed operator is $\sqrt{-\Delta}$ by writing $(1.2)$ as a system of order 1 . At this time, a serious difficulty is that the frequencies of $\sqrt{-\Delta}$, still denoted by $\lambda_{k}(k \in \mathbb{Z})$, have no good separation property and $\left|\lambda_{k}-\lambda_{k^{\prime}}\right|$ is dense, at least, in some interval of $\mathbb{R}$, when $d>1$. Thus, the reducibility for 1.2 with $d>1$ is a challenging open problem. See 24 for recent progress. However, the reducibility for 1.2 with $d=1$ can be derived from the earlier KAM theorem (see [20] and 25]) for nonlinear partial differential equations, assuming $V$ is analytic in $(t, x)$. Also see, 21.

According to our knowledge, the reducibility for 1.2 with perturbation of finite smoothness has not been treated explicitly in the literatures. Usually, the spectrum property of operators depends heavily on the smoothness of the perturbation. For example, the Anderson localization and positivity of the Lyapunov exponent for one frequency discrete quasi-period Schrödinger operator with analytic potential occur in non-perturbative sense (the largeness of the potential does not depend on the Diophantine condition. See [8], for the detail). However, one can only get perturbative results when the analytic property of the potential is weakened to Gevrey regularity (see [19]). Thus, the reducibility is worth studying when the perturbation $V$ is of finite smoothness in $(t, x)$. We also mention the papers by Baldi-Berti-Montalto [1] for KdV equation and Feola-Procesi [15] for nonlinear Schrödinger equation where the reducibility is obtained in the finite differentiable case (using "tame" estimates on Sobolev spaces) and in the case of unbounded nonlinearities.

Let us consider a linear wave equation with quasi-periodic coefficient:

$$
u_{t t}-u_{x x}+\varepsilon V(\omega t, x) u=0
$$

subject to the boundary condition

$$
u(t,-\pi)=u(t, \pi)=0 .
$$


For $p \geq 0$, let $\mathscr{H}^{p}[-\pi, \pi]$ be the usual Sobolev space. Define

$$
\mathscr{H}_{0}^{p}[-\pi, \pi]=\left\{u \in \mathscr{H}^{p}[-\pi, \pi]: \int_{-\pi}^{\pi} u(x) d x=0, u(-\pi)=u(\pi)=0\right\} .
$$

Assumption A. Assume $V$ is a $C^{N}$-smooth and quasi-periodic in time $t$ with frequency $\omega \in \mathbb{R}^{n}:$ that is, there is a hull function $\mathscr{V}(\theta, x) \in C^{N}\left(\mathbb{T}^{n} \times[-\pi, \pi], \mathbb{R}\right)$ such that

$$
V(\omega t, x)=\left.\mathscr{V}(\theta, x)\right|_{\theta=\omega t}, \quad \mathbb{T}^{n}=\mathbb{R}^{n} / 2 \pi \mathbb{Z}^{n},
$$

where $N>200 n$.

Assumption B. We also assume that $V$ is an even function of $x$, with zero-average:

$$
\int_{-\pi}^{\pi} V(\omega t, x) d x \equiv 0
$$

Assumption C. Assume $\omega=\tau \omega_{0}$, where $\omega_{0}$ is Diophantine:

$$
\left|\left\langle k, \omega_{0}\right\rangle\right| \geq \frac{\gamma}{|k|^{n+1}}, \quad k \in \mathbb{Z}^{n} \backslash\{0\},
$$

where $\gamma$ is a constant and $0<\gamma \ll 1, \tau \in[1,2]$ is a parameter.

Let $w=u_{t}$. Endow $L^{2}[-\pi, \pi] \times L^{2}[-\pi, \pi]$ with the symplectic form $d w \wedge d u$. Take $\left(L^{2}[-\pi, \pi] \times L^{2}[-\pi, \pi], d w \wedge d u\right)$ as phase space. Then $[1.3)$ is a hamiltonian system with hamiltonian functional

$$
H(u, w)=\int_{-\pi}^{\pi}\left(\frac{1}{2}\left(w^{2}+u_{x}^{2}\right)+\frac{1}{2} \varepsilon V(\omega t, x) u^{2}\right) d x
$$

and the Hamiltonian equation

$$
u_{t}=\frac{\delta H}{\delta w}, \quad w_{t}=-\frac{\delta H}{\delta u}
$$

With these three assumptions, we can describe the main results of this paper.

Theorem 1.1. With Assumptions A, B, C, for given $1 \gg \gamma>0$, there exists $\varepsilon^{*}$ with $0<\varepsilon^{*}=\varepsilon^{*}(n, \gamma) \ll \gamma$, and exists a subset $\Pi \subset[1,2]$ with

$$
\operatorname{mes} \Pi \geq 1-O\left(\gamma^{1 / 3}\right)
$$

such that for any $0<\varepsilon<\varepsilon^{*}$ and for any $\tau \in \Pi$, there is a quasi-periodic symplectic change $u=\left.\Phi(\theta, x) v\right|_{\theta=\omega t}$ with the map $\theta \mapsto \Phi(\theta, \cdot)$ being of class $C^{N-\mu}\left(\mathbb{T}^{n}, L\left(\mathscr{H}_{0}^{N}[-\pi, \pi]\right.\right.$, $\left.\left.\mathscr{H}_{0}^{N}[-\pi, \pi]\right)\right)$ for any $\mu \in(0,1)$ and satisfying

$$
\|\Phi(\theta, \cdot)-\mathrm{id}\|_{L\left(\mathscr{H}_{0}^{N}[-\pi, \pi], \mathscr{H}_{0}^{N}[-\pi, \pi]\right)} \leq C_{\mu} \varepsilon
$$


where id is the identity from $\mathscr{H}_{0}^{N}[-\pi, \pi] \rightarrow \mathscr{H}_{0}^{N}[-\pi, \pi], C_{\mu}$ is a constant depending $\mu$ and $L\left(\mathscr{H}_{0}^{N}[-\pi, \pi], \mathscr{H}_{0}^{N}[-\pi, \pi]\right)$ is the class of all bounded linear operators from $\mathscr{H}_{0}^{N}[-\pi, \pi]$ to itself which changes (1.3) subject to (1.4) into

$$
v_{t t}-v_{x x}+\varepsilon M_{\xi} v=0, \quad v(t,-\pi)=v(t, \pi)=0,
$$

where $M_{\xi}$ is a real Fourier multiplier:

$$
M_{\xi} \sin k x=\xi_{k} \sin k x, \quad k \in \mathbb{N}
$$

with constants $\xi_{k} \in \mathbb{R}$ and $\left|\xi_{k}\right| \leq C /|k|$, where $C$ is an absolute constant, mes $\Pi$ denotes Lebesgue measure for set $\Pi$.

Corollary 1.2. With Assumptions A, B, C, for any $\tau \in \Pi$ and $0<\varepsilon<\varepsilon^{*}$, the wave operator

$$
\mathscr{L} u(t, x)=\left(\partial_{t}^{2}-\partial_{x}^{2}+\varepsilon V(\omega t, x)\right) u(t, x), \quad u(t,-\pi)=u(t, \pi)=0
$$

is of pure point spectrum property and of zero Lyapunov exponent.

Corollary 1.3. With Assumptions A, B, C, for any $\tau \in \Pi$ and $0<\varepsilon<\varepsilon^{*}$, the initial problem of the linear wave equation

$$
\left(\partial_{t}^{2}-\partial_{x}^{2}+\varepsilon V(\omega t, x)\right) u(t, x)=0, \quad u(0, x)=u_{0}(x), \quad u_{t}(0, x)=\widetilde{u}_{0}(x)
$$

with $\left(u_{0}, \widetilde{u}_{0}\right) \in \mathscr{H}_{0}^{N}[-\pi, \pi] \times \mathscr{H}_{0}^{N-1}[-\pi, \pi]$ has a unique solution $u(t, x)$ which is almost periodic in time and obeys the following estimate

$$
\begin{aligned}
& (1-c \sqrt{\varepsilon})\left(\left\|u_{0}\right\|_{\mathscr{H}^{N}}^{2}+\left\|\widetilde{u}_{0}\right\|_{\mathscr{H}^{N-1}}^{2}\right) \leq\|u(t)\|_{\mathscr{H}^{N}}^{2} \leq(1+c \sqrt{\varepsilon})\left(\left\|u_{0}\right\|_{\mathscr{H}^{N}}^{2}+\left\|\widetilde{u}_{0}\right\|_{\mathscr{H}^{N-1}}^{2}\right), \\
& (1-c \sqrt{\varepsilon})\left(\left\|u_{0}\right\|_{\mathscr{H}^{N}}^{2}+\left\|\widetilde{u}_{0}\right\|_{\mathscr{H}^{N-1}}^{2}\right) \leq\left\|u_{t}(t)\right\|_{\mathscr{H}^{N-1}}^{2} \leq(1+c \sqrt{\varepsilon})\left(\left\|u_{0}\right\|_{\mathscr{H}^{N}}^{2}+\left\|\widetilde{u}_{0}\right\|_{\mathscr{H}^{N-1}}^{2}\right),
\end{aligned}
$$

where $c$ is a positive constant which might be different in different places.

Remark 1.4. In references [7,23], there are solutions $u(t, x)$ obey

$$
\|u(t)\|_{\mathscr{H}^{N}}=C(\log |t|)^{C} \rightarrow \infty \quad \text { as }|t| \rightarrow \infty
$$

for the nonlinear Schrödinger equation (1.1]. In [21], it is proved that for the following linear wave equation

$$
u_{t t}-u_{x x}+M u-\varepsilon(\cos 2 t) u=0
$$

subject to Dirichlet boundary condition on $[0, \pi]$, there is a solution $u(t, x)$ obeying

$$
\left\|u\left(t_{j}\right)\right\|_{\mathscr{H}^{1}(\mathbb{T})} \rightarrow \infty, \quad\left|t_{j}\right| \rightarrow \infty
$$

The above results show that one can not avoid restricting the choice of parameters $\omega$ (or $\tau)$ to a Cantor type subset $\Pi$ of the parameter set $[1,2]$ in Corollary 1.3 . 
Remark 1.5. In [5], it is proved that there is a quasi-periodic solution for $d$-dimensional nonlinear wave equation with a quasi-periodic in time nonlinearity like

$$
u_{t t}-\Delta u-V(x) u=\varepsilon f(\omega t, x, u), \quad x \in \mathbb{T}^{d},
$$

where the multiplicative potential $V$ is in $C^{q}\left(\mathbb{T}^{d} ; \mathbb{R}\right), \omega \in \mathbb{R}^{n}$ is a non-resonant frequency vector and $f \in C^{q}\left(\mathbb{T}^{n} \times \mathbb{T}^{d} \times \mathbb{R} ; \mathbb{R}\right)$. Because of the application of Nash-Moser iteration, it is not clear whether the obtained quasi-periodic solution is linearly stable or has zero Lyapunov exponent. As a corollary of Theorem 1.1, we can prove that the quasi-periodic solution by 5 is linearly stable and has zero Lyapunov exponent, when $d=1$.

Remark 1.6. As mentioned above, the reducibility of the linear wave equation with time quasi-periodic and analytic coefficients can be implicitly derived from the KAM theory dealing with the existence of KAM tori for nonlinear wave equation. Here we should note the difference between the analytic coefficient and the finitely smooth one. By passing to Fourier coefficients, the wave equation 1.3 can be written as a linear Hamiltonian system with Hamiltonian

$$
H=\langle\Lambda z, \bar{z}\rangle+\varepsilon\left[\left\langle R^{z z}(\theta) z, z\right\rangle+\left\langle R^{z \bar{z}}(\theta) z, \bar{z}\right\rangle+\left\langle R^{\overline{z z}}(\theta) \bar{z}, \bar{z}\right\rangle\right]
$$

where the symplectic form is $\mathbf{i} d z \wedge d \bar{z}$. The basic task is to search a series of symplectic coordinate changes to eliminate the perturbations $R^{z z}(\theta), R^{z \bar{z}}(\theta)$ and $R^{\overline{z z}}(\theta)$ except for the averages of the diagonal terms of $R^{z \bar{z}}(\theta)$. To this end, the symplectic coordinate changes are the time- 1 map of the flow for the Hamiltonian $\varepsilon F$, where $F$ is of the form

$$
F=\left\langle F^{z z}(\theta, \tau) z, z\right\rangle+\left\langle F^{z \bar{z}}(\theta, \tau) z, \bar{z}\right\rangle+\left\langle F^{\overline{z z}}(\theta, \tau) \bar{z}, \bar{z}\right\rangle .
$$

- When the potential $V(\theta)(\theta=\omega t)$ is analytic in some strip domain $|\operatorname{Im} \theta| \leq s_{\nu}^{*}$, (where $\nu$ is the KAM iteration step), the perturbations $R^{z z}(\theta), R^{z \bar{z}}(\theta)$ and $R^{\overline{z z}}(\theta)$ are also analytic in $|\operatorname{Im} \theta| \leq s_{\nu}^{*}$. An important fact in this analytic case is that $s_{\nu}^{*}$ 's have a uniform non-zero below bound:

$$
s_{\nu}^{*} \geq \frac{s_{0}}{2}, \quad s_{0}>0 \quad \text { for all } \nu=1,2, \ldots
$$

- When the potential $V(\theta)$ is finitely smooth of order $N$, by using Jackson-MoserZehnder approximate lemma, we can still make sure that $R^{z z}(\theta), R^{z \bar{z}}(\theta)$ and $R^{\overline{z z}}(\theta)$ are analytic in $|\operatorname{Im} \theta| \leq s_{\nu}$ at the $\nu$-th KAM step. However, the strip width $s_{\nu}$ 's have no non-zero below bound. Actually, $s_{\nu}$ goes to zero very rapidly:

$$
s_{\nu}=\varepsilon_{\nu+1}^{1 / N}, \quad \varepsilon_{\nu}=\varepsilon^{(4 / 3)^{\nu}}, \quad \nu=1,2, \ldots
$$

- For analytic case, we can prove the Hamiltonian $\varepsilon F=O\left(\varepsilon_{\nu}\right)$ at the $\nu$-th KAM step, because of $s_{\nu}^{*} \geq s_{0} / 2$. It follows immediately that the new perturbation is $\{\varepsilon F, \varepsilon R\}=O\left(\varepsilon_{\nu}^{2}\right)=O\left(\varepsilon_{\nu+1}\right)$. 
- For the finitely smooth case, the situation is much more complicated. At this case, we find $\varepsilon F=O\left(\varepsilon_{\nu}^{1-6(n+1) / N}\right)$ at the $\nu$-th KAM step. Thus, for the finitely smooth potential $V \in C^{N}$, the new perturbation is $\{\varepsilon F, \varepsilon R\}=O\left(\varepsilon_{\nu}^{2-6(n+1) / N}\right)$. In order to guarantee the quadratic convergence of the KAM iterations, that is, $O\left(\varepsilon_{\nu}^{2-6(n+1) / N}\right)=O\left(\varepsilon_{\nu}^{4 / 3}\right)=O\left(\varepsilon_{\nu+1}\right)$, it is necessary to assume the smoothness order $N \gg 1$. It is enough to assume $N>200 n$. Clearly, this is not sharp. In this paper, we do not pursue the lowest smoothness for the potential $V$.

Finally, we list some related results:

In [3], Bambusi and Graffi eliminated by KAM methods the time dependence in 1dimensional Schrödinger equation

$$
H(t) \psi(x, t)=\mathbf{i} \partial_{t} \psi(x, t), \quad x \in \mathbb{R} ; \quad H(t):=-\frac{d^{2}}{d x^{2}}+Q(x)+\varepsilon V(x, \omega t), \quad \varepsilon \in \mathbb{R},
$$

where $Q(x) \in C^{\infty}(\mathbb{R} ; \mathbb{R}), Q(x) \sim|x|^{\alpha}$ for $\alpha>2$ as $|x| \rightarrow \infty$ and $V(x, \phi)$ is a $C^{\infty}(\mathbb{R} ; \mathbb{R})$ valued holomorphic function of $\phi \in \mathbb{T}^{n}$, with $|V(x, \phi)||x|^{-\beta}$ bounded as $|x| \rightarrow \infty$ for some $\beta<(\alpha-2) / 2$. The proof is based on Kuksin's estimate of solutions of homological equations with nonconstant coefficients. When $\alpha>2$ and $\beta=(\alpha-2) / 2$, the methods used in [3] will become invalid. Afterward, Liu and Yuan [22] solved this case by a new estimate for the solution of the homological equation. Wang 29] proved the pure-point nature of the spectrum of the Floquet operator $K_{F}$ :

$$
K_{F}=-\mathbf{i} \sum_{k=1}^{n} \omega_{k} \frac{\partial}{\partial \theta_{k}}-\frac{d^{2}}{d x^{2}}+x^{2}+\varepsilon V(\theta, x) .
$$

The spectral properties of the Floquet operator $K_{F}$ is closely related to the long-time behavior of the solution $\psi(t, x)$ of the equation (1.7) with $Q(x)=x^{2}$. The author considered $V(x, \theta)=e^{-x^{2}} \sum_{k=1}^{n} \cos \theta_{k}$, which has exponential decay. The case $\beta<(\alpha-2) / 2=0$ was solved by Grébert and Thomann [17], where $V(x, \theta)$ has polynomial decay. In 30 the above results were improved, in which $V(x, \theta)$ has logarithmic decay.

Grébert and Paturel [16] proved that a linear $d$-dimensional Schrödinger equation on $\mathbb{R}^{d}$ with harmonic potential $|x|^{2}$ and small $t$-quasiperiodic potential

$$
\mathbf{i} \partial_{t} u-\Delta u+|x|^{2} u+\varepsilon V(t \omega, x) u=0, \quad x \in \mathbb{R}^{d}
$$

reduces to an autonomous system for most values of the frequency vector $\omega \in \mathbb{R}^{n}$.

In [14], Fang, Han and Wang proved Anderson localization for the Klein-Gordon operator under non-resonant perturbations. The authors showed that the Sobolev norms of solutions to the corresponding Klein-Gordon equations remain bounded for all time.

Remark 1.7. In [28], it is proved that the wave equation of time quasi-periodic coefficients

$$
u_{t t}-u_{x x}+M u+\varepsilon\left(V_{0}(\omega t) u_{x x}+V(\omega t, x) u\right)=0
$$


subject to periodic boundary condition $x \in \mathbb{T}=\mathbb{R} / 2 \pi \mathbb{Z}$ can be reduced by a time quasiperiodic symplectic change to a linear Hamiltonian system of constant coefficients

$$
\dot{\widetilde{q}}=(\Lambda+\varepsilon \widetilde{Q}) \widetilde{p}, \quad \dot{\widetilde{p}}=-(\Lambda+\varepsilon \widetilde{Q}) \widetilde{q}
$$

where $\Lambda=\operatorname{diag}\left(\Lambda_{j}: j=0,1,2, \ldots\right), \Lambda_{0}=\rho \sqrt{M}, \Lambda_{j}=\rho \sqrt{j^{2}+M} E_{22}, \rho$ is a constant close to $1, E_{22}$ is a $2 \times 2$ unit matrix, and $\widetilde{Q}=\operatorname{diag}\left(\widetilde{Q}_{i}: i=0,1,2, \ldots\right)$ is independent of time with $\widetilde{Q}_{0} \in \mathbb{R}, \widetilde{Q}_{i}$ being a real $2 \times 2$ matrix, and $\left|\widetilde{Q}_{i}\right| \leq C / i, i=1,2, \ldots$

Since the eigenvalues of the differential operator $-\partial_{x x}$ with the periodic boundary condition $x \in \mathbb{T}=\mathbb{R} / 2 \pi \mathbb{Z}$ possesses multiplicity 2 , the reduced linear operator $\Lambda+\varepsilon \widetilde{Q}$ is not diagonal, although it is block diagonal. Thus $(1.8)$ can not be written as a linear wave equation with Fourier multiplier $M_{\xi}$ :

$$
u_{t t}-u_{x x}+M_{\xi} u=0 .
$$

In the present paper, the eigenvalues of the differential operator $-\partial_{x x}$ with Dirichlet boundary condition are simple. By this fact, we can reduce the wave equation

$$
u_{t t}-u_{x x}+\varepsilon V(\omega t, x) u=0
$$

with boundary condition

$$
u(t,-\pi)=u(t, \pi)=0
$$

to a new equation with Fourier multiplier $M_{\xi}$ :

$$
v_{t t}-v_{x x}+\varepsilon M_{\xi} v=0 .
$$

\section{Passing to Fourier coefficients}

Consider the differential equation

$$
\mathscr{L} u=u_{t t}-u_{x x}+\varepsilon V(\omega t, x) u=0
$$

subject to the boundary condition

$$
u(t,-\pi)=u(t, \pi)=0 .
$$

It is well-known that the Sturm-Liouville problem

$$
-y^{\prime \prime}=\lambda y
$$

with the boundary condition

$$
y(-\pi)=y(\pi)=0
$$


has the eigenvalues and eigenfunctions, respectively,

$$
\begin{aligned}
\lambda_{k} & =k^{2}, & k & =1,2, \ldots, \\
\phi_{k}(x) & =\sin k x, & k & =1,2, \ldots
\end{aligned}
$$

Make the ansatz

$$
u(t, x)=\mathscr{S}\left(u_{k}\right)=\sum_{k=1}^{\infty} u_{k}(t) \phi_{k}(x) .
$$

Note that $V$ is an even function of $x$ such that $\int_{-\pi}^{\pi} V(\omega t, x) d x \equiv 0$. Write

$$
V(\omega t, x)=\sum_{k=1}^{\infty} v_{k}(\omega t) \varphi_{k}(x)
$$

where $\varphi_{k}(x)=\cos k x, k=1,2, \ldots$ Let

$$
\frac{d u_{k}}{d t}=w_{k}
$$

By the fact that

$$
\varphi_{j} \phi_{l}=\sum_{k=1}^{\infty}\left\langle\varphi_{j} \phi_{l}, \phi_{k}\right\rangle \phi_{k}, \quad j, l=1,2, \ldots,
$$

then 2.1 can be expressed as

$$
\sum_{k=1}^{\infty}\left(\frac{d w_{k}}{d t}+\lambda_{k} u_{k}+\varepsilon \sum_{l=1}^{\infty} \sum_{j=1}^{\infty} c_{j l k} v_{j} u_{l}\right) \phi_{k}=0
$$

which implies that

$$
\frac{d w_{k}}{d t}=-\lambda_{k} u_{k}-\varepsilon \sum_{l=1}^{\infty} \sum_{j=1}^{\infty} c_{j l k} v_{j} u_{l}
$$

where

$$
c_{j l k}=\left\langle\varphi_{j} \phi_{l}, \phi_{k}\right\rangle=\int_{-\pi}^{\pi} \cos j x \cdot \sin l x \cdot \sin k x d x= \begin{cases}0 & \text { if } k \neq \pm l \pm j \\ \pi / 2 & \text { if } k=l \pm j \geq 1 \\ -\pi / 2 & \text { if } k=-l \pm j \geq 1\end{cases}
$$

Rescale

$$
\mathscr{T}: \quad w_{k}=\sqrt[4]{\lambda_{k}} p_{k}, \quad u_{k}=\frac{1}{\sqrt[4]{\lambda_{k}}} q_{k}
$$

Then

$$
\dot{q}_{k}=\sqrt{\lambda_{k}} p_{k}, \quad \dot{p}_{k}=-\sqrt{\lambda_{k}} q_{k}-\varepsilon \sum_{l=1}^{\infty} \sum_{j=1}^{\infty} c_{j l k} \frac{v_{j}(\theta)}{\sqrt[4]{\lambda_{k} \lambda_{l}}} q_{l} .
$$


This is a linear Hamiltonian system

$$
\dot{q}_{k}=\frac{\partial H}{\partial p_{k}}, \quad \dot{p}_{k}=-\frac{\partial H}{\partial q_{k}},
$$

where the symplectic structure is $d p \wedge d q=\sum_{j=1}^{\infty} d p_{j} \wedge d q_{j}$ and the Hamiltonian function is

$$
H(p, q)=\sum_{k=1}^{\infty} \frac{\sqrt{\lambda_{k}}\left(p_{k}^{2}+q_{k}^{2}\right)}{2}+\varepsilon \sum_{k=1}^{\infty} \sum_{l=1}^{\infty} \sum_{j=1}^{\infty} c_{j l k} \frac{v_{j}(\theta)}{\sqrt[4]{\lambda_{k} \lambda_{l}}} q_{l} q_{k}, \quad \theta=\omega t .
$$

Introduce complex variables:

$$
z_{j}=\frac{1}{\sqrt{2}}\left(q_{j}-\mathbf{i} p_{j}\right), \quad \bar{z}_{j}=\frac{1}{\sqrt{2}}\left(q_{j}+\mathbf{i} p_{j}\right),
$$

which is a symplectic transformation with $d p \wedge d q=\mathbf{i} d z \wedge d \bar{z}$. Thus (2.4) is changed into

$$
\mathscr{G}: \quad \dot{z}_{k}=\mathbf{i} \frac{\partial H}{\partial \bar{z}_{k}}, \quad \dot{\bar{z}}_{k}=-\mathbf{i} \frac{\partial H}{\partial z_{k}}
$$

where

$$
H(z, \bar{z})=\sum_{k=1}^{\infty} \sqrt{\lambda_{k}} z_{k} \bar{z}_{k}+\varepsilon \sum_{k=1}^{\infty} \sum_{l=1}^{\infty} \sum_{j=1}^{\infty} c_{j l k} \frac{v_{j}(\theta)}{\sqrt[4]{\lambda_{k} \lambda_{l}}}\left(\frac{z_{l}+\bar{z}_{l}}{\sqrt{2}}\right)\left(\frac{z_{k}+\bar{z}_{k}}{\sqrt{2}}\right) .
$$

For two sequences $x=\left(x_{j} \in \mathbb{C}, j=1,2, \ldots\right), y=\left(y_{j} \in \mathbb{C}, j=1,2, \ldots\right)$, define

$$
\langle x, y\rangle=\sum_{j=1}^{\infty} x_{j} y_{j}
$$

Then we can write

$$
H=\langle\Lambda z, \bar{z}\rangle+\varepsilon\left[\left\langle R^{z z}(\theta) z, z\right\rangle+\left\langle R^{z \bar{z}}(\theta) z, \bar{z}\right\rangle+\left\langle R^{\overline{z z}}(\theta) \bar{z}, \bar{z}\right\rangle\right]
$$

where

$$
\begin{gathered}
\Lambda=\operatorname{diag}\left(\sqrt{\lambda_{j}}: j=1,2, \ldots\right), \quad \theta=\omega t \\
R^{z z}(\theta)=\left(R_{k l}^{z z}(\theta): k, l=1,2, \ldots\right), \quad R_{k l}^{z z}(\theta)=\frac{1}{2} \sum_{j=1}^{\infty} \frac{c_{j l k} v_{j}(\theta)}{\sqrt[4]{\lambda_{k}} \sqrt[4]{\lambda_{l}}} \\
R^{z \bar{z}}(\theta)=\left(R_{k l}^{z \bar{z}}(\theta): k, l=1,2, \ldots\right), \quad R_{k l}^{z \bar{z}}(\theta)=\sum_{j=1}^{\infty} \frac{c_{j l k} v_{j}(\theta)}{\sqrt[4]{\lambda_{k}} \sqrt[4]{\lambda_{l}}} \\
R^{\overline{z z}}(\theta)=\left(R_{k l}^{\overline{z z}}(\theta): k, l=1,2, \ldots\right), \quad R_{k l}^{\overline{z z}}(\theta)=\frac{1}{2} \sum_{j=1}^{\infty} \frac{c_{j l k} v_{j}(\theta)}{\sqrt[4]{\lambda_{k}} \sqrt[4]{\lambda_{l}}}
\end{gathered}
$$

Define a Hilbert space $h_{N}$ as follows:

$$
h_{N}=\left\{z=\left(z_{k} \in \mathbb{C}: k=1,2, \ldots\right)\right\}
$$


Let

$$
\langle y, z\rangle_{N}:=\sum_{k=1}^{\infty} k^{2 N} y_{k} \bar{z}_{k}, \quad \forall y, z \in h_{N}, \quad \text { and } \quad\|z\|_{N}^{2}=\langle z, z\rangle_{N}
$$

Recall that

$$
\mathscr{V}(\theta, x) \in C^{N}\left(\mathbb{T}^{n} \times[-\pi, \pi], \mathbb{R}\right) .
$$

Note that the Fourier transformation 2.2$)$ is isometric from $u \in \mathscr{H}^{N}[-\pi, \pi]$ to $\left(u_{k}: k=\right.$ $1,2, \ldots) \in h_{N}$, where $\mathscr{H}^{N}[-\pi, \pi]$ is the usual Sobolev space.

Now we need the following lemmas.

\section{Lemma 2.1.}

$$
\begin{aligned}
& \sup _{\theta \in \mathbb{T}^{n}}\left\|\sum_{|\alpha|=N} \partial_{\theta}^{\alpha} J R^{z z}(\theta) J\right\|_{h_{N} \rightarrow h_{N}} \leq C, \\
& \sup _{\theta \in \mathbb{T}^{n}}\left\|\sum_{|\alpha|=N} \partial_{\theta}^{\alpha} J R^{z \bar{z}}(\theta) J\right\|_{h_{N} \rightarrow h_{N}} \leq C, \\
& \sup _{\theta \in \mathbb{T}^{n}}\left\|\sum_{|\alpha|=N} \partial_{\theta}^{\alpha} J R^{\overline{z z}}(\theta) J\right\|_{h_{N} \rightarrow h_{N}} \leq C,
\end{aligned}
$$

where $\|\cdot\|_{h_{N} \rightarrow h_{N}}$ is the operator norm from $h_{N}$ to $h_{N}$, and $\alpha=\left(\alpha_{1}, \alpha_{2}, \ldots, \alpha_{n}\right),|\alpha|=$ $\left|\alpha_{1}\right|+\left|\alpha_{2}\right|+\cdots+\left|\alpha_{n}\right|, \alpha_{j}$ 's are positive integers, and $J=\operatorname{diag}\left(\sqrt[4]{\lambda_{j}}: j=1,2, \ldots\right)=$ $\operatorname{diag}(\sqrt{j}: j=1,2, \ldots)$.

Proof. By (2.5),

$$
\partial_{\theta}^{\alpha} J R^{z z}(\theta) J=\left(\frac{1}{2} \sum_{j=1}^{\infty} C_{j l k} \partial_{\theta}^{\alpha} v_{j}(\theta): l, k=1,2, \ldots\right),
$$

where $C_{j l k}$ is defined as 2.3 . For any $z=\left(z_{k} \in \mathbb{C}: k=1,2, \ldots\right) \in h_{N}$,

$$
\left(\sum_{|\alpha|=N} \partial_{\theta}^{\alpha} J R^{z z}(\theta) J\right) z=\left(\frac{1}{2} \sum_{j=1}^{\infty} \sum_{k=1}^{\infty} C_{j l k}\left(\sum_{|\alpha|=N} \partial_{\theta}^{\alpha} v_{j}(\theta)\right) z_{k}: l=1,2, \ldots\right) .
$$

Let

$$
\gamma_{l j}=\frac{( \pm l \pm j) j}{l}, \quad \text { where }( \pm l \pm j) j l \neq 0
$$

Thus,

$$
\begin{aligned}
& \left\|\left(\sum_{|\alpha|=N} \partial_{\theta}^{\alpha} J R^{z z}(\theta) J\right) z\right\|_{N}^{2} \\
= & \sum_{l=1}^{\infty} l^{2 N}\left|\frac{1}{2} \sum_{j=1}^{\infty} \sum_{k=1}^{\infty} C_{j l k}\left(\sum_{|\alpha|=N} \partial_{\theta}^{\alpha} v_{j}(\theta)\right) z_{k}\right|^{2}
\end{aligned}
$$




$$
\begin{aligned}
& =\sum_{l=1}^{\infty} l^{2 N}\left|\frac{1}{2} \sum_{j=1}^{\infty} C_{j l( \pm l \pm j)}\left(\sum_{|\alpha|=N} \partial_{\theta}^{\alpha} v_{j}(\theta)\right) z_{ \pm l \pm j}\right|^{2} \\
& =\frac{1}{4} \sum_{l=1}^{\infty} l^{2 N}\left|\sum_{j=1}^{\infty} \frac{1}{\gamma_{l j}^{N}} \cdot \gamma_{l j}^{N} C_{j l( \pm l \pm j)}\left(\sum_{|\alpha|=N} \partial_{\theta}^{\alpha} v_{j}(\theta)\right) z_{ \pm l \pm j}\right|^{2} \\
& \leq C \sum_{l=1}^{\infty}\left(\sum_{j=1}^{\infty} \frac{1}{\gamma_{l j}^{2 N}}\right)\left(\sum_{j=1}^{\infty}|j|^{2 N}\left|\sum_{|\alpha|=N} \partial_{\theta}^{\alpha} v_{j}(\theta)\right|^{2}| \pm l \pm j|^{2 N}\left|z_{ \pm l \pm j}\right|^{2}\right) \\
& \leq C\left(\sum_{j=1}^{\infty}|j|^{2 N}\left|\sum_{|\alpha|=N} \partial_{\theta}^{\alpha} v_{j}(\theta)\right|^{2} \sum_{l=1}^{\infty}| \pm l \pm j|^{2 N}\left|z_{ \pm l \pm j}\right|^{2}\right) \\
& \leq C \sum_{j=1}^{\infty}|j|^{2 N}\left|\sum_{|\alpha|=N} \partial_{\theta}^{\alpha} v_{j}(\theta)\right|^{2}\|z\|_{N}^{2} \\
& \leq C \sup _{(\theta, x) \in \mathbb{T}^{n} \times[-\pi, \pi]}\left|\sum_{|\alpha|=N} \partial_{\theta}^{\alpha} \partial_{x}^{N} \mathscr{V}(\theta, x)\right|\|z\|_{N}^{2} \leq C\|z\|_{N}^{2},
\end{aligned}
$$

where $C$ is a universal constant which might be different in different places. It follows

$$
\sup _{\theta \in \mathbb{T}^{n}}\left\|\sum_{|\alpha|=N} \partial_{\theta}^{\alpha} J R^{z z}(\theta) J\right\|_{h_{N} \rightarrow h_{N}} \leq C .
$$

The proofs of the last two inequalities in (2.6) are similar to that of 2.7.

\section{Analytical approximation lemma}

We need to find a series of operators which are analytic in some complex strip domains to approximate the operators $R^{z z}(\theta), R^{z \bar{z}}(\theta)$ and $R^{\overline{z z}}(\theta)$. To this end, we use an approximation lemma developed in 18, 26, 27]. This method is used in [31, too.

We start by recalling some definitions and setting some new notations. Assume $X$ is a Banach space with the norm $\|\cdot\|_{X}$. First recall that $C^{\mu}\left(\mathbb{R}^{n} ; X\right)$ for $0<\mu<1$ denotes the space of bounded Hölder continuous functions $f: \mathbb{R}^{n} \mapsto X$ with the form

$$
\|f\|_{C^{\mu}, X}=\sup _{0<|x-y|<1} \frac{\|f(x)-f(y)\|_{X}}{|x-y|^{\mu}}+\sup _{x \in \mathbb{R}^{n}}\|f(x)\|_{X} .
$$

If $\mu=0$ then $\|f\|_{C^{\mu}, X}$ denotes the sup-norm. For $\ell=k+\mu$ with $k \in \mathbb{N}$ and $0 \leq \mu<1$, we denote by $C^{\ell}\left(\mathbb{R}^{n} ; X\right)$ the space of functions $f: \mathbb{R}^{n} \mapsto X$ with Hölder continuous partial derivatives, i.e., $\partial^{\alpha} f \in C^{\mu}\left(\mathbb{R}^{n} ; X_{\alpha}\right)$ for all multi-indices $\alpha=\left(\alpha_{1}, \ldots, \alpha_{n}\right) \in \mathbb{N}^{n}$ with the assumption that $|\alpha|:=\left|\alpha_{1}\right|+\cdots+\left|\alpha_{n}\right| \leq k$ and $X_{\alpha}$ is the Banach space of bounded operators $T: \prod^{|\alpha|}\left(\mathbb{R}^{n}\right) \mapsto X$ with the norm

$$
\|T\|_{X_{\alpha}}=\sup \left\{\left\|T\left(u_{1}, u_{2}, \ldots, u_{|\alpha|}\right)\right\|_{X}:\left\|u_{i}\right\|=1,1 \leq i \leq|\alpha|\right\} .
$$


We define the norm

$$
\|f\|_{C^{\ell}}=\sup _{|\alpha| \leq \ell}\left\|\partial^{\alpha} f\right\|_{C^{\mu}, X_{\alpha}}
$$

Lemma 3.1 (Jackson-Moser-Zehnder). Let $f \in C^{\ell}\left(\mathbb{R}^{n} ; X\right)$ for some $\ell>0$ with finite $C^{\ell}$ norm over $\mathbb{R}^{n}$. Let $\phi$ be a radial-symmetric, $C^{\infty}$ function, having as supporting the closure of the unit ball centered at the origin, where $\phi$ is completely flat and takes value 1 , let $K=\widehat{\phi}$ be its Fourier transform. For all $\sigma>0$ define

$$
f_{\sigma}(x):=K_{\sigma} * f=\frac{1}{\sigma^{n}} \int_{\mathbb{R}^{n}} K\left(\frac{x-y}{\sigma}\right) f(y) d y .
$$

Then there exists a constant $C \geq 1$ depending only on $\ell$ and $n$ such that the following holds: For any $\sigma>0$, the function $f_{\sigma}(x)$ is a real-analytic function from $\mathbb{C}^{n} /(\pi \mathbb{Z})^{n}$ to $X$ such that if $\Delta_{\sigma}^{n}$ denotes the $n$-dimensional complex strip of width $\sigma$,

$$
\Delta_{\sigma}^{n}:=\left\{x \in \mathbb{C}^{n}|| \operatorname{Im} x_{j} \mid \leq \sigma, 1 \leq j \leq n\right\}
$$

then $\forall \alpha \in \mathbb{N}^{n}$ such that $|\alpha| \leq \ell$ one has

$$
\sup _{x \in \Delta_{\sigma}^{n}}\left\|\partial^{\alpha} f_{\sigma}(x)-\sum_{|\beta| \leq \ell-|\alpha|} \frac{\partial^{\beta+\alpha} f(\operatorname{Re} x)}{\beta !}(\mathbf{i} \operatorname{Im} x)^{\beta}\right\|_{X_{\alpha}} \leq C\|f\|_{C^{\ell}} \sigma^{\ell-|\alpha|},
$$

and for all $0 \leq s \leq \sigma$,

$$
\sup _{x \in \Delta_{s}^{n}}\left\|\partial^{\alpha} f_{\sigma}(x)-\partial^{\alpha} f_{s}(x)\right\|_{X_{\alpha}} \leq C\|f\|_{C^{\ell}} \sigma^{\ell-|\alpha|} .
$$

The function $f_{\sigma}$ preserves periodicity (i.e., if $f$ is T-periodic in any of its variable $x_{j}$, so is $\left.f_{\sigma}\right)$. Finally, if $f$ depends on some parameter $\xi \in \Pi \subset \mathbb{R}^{n}$ and if

$$
\|f(x, \xi)\|_{C^{\ell}(X)}^{\mathscr{L}}:=\sup _{\xi \in \Pi}\left\|\partial_{\xi} f(x, \xi)\right\|_{C^{\ell}(X)}
$$

are uniformly bounded by a constant $C$ then all the above estimates hold with $\|\cdot\|$ replaced by $\|\cdot\|^{\mathscr{L}}$.

The proof of this lemma consists in a direct check which is based on standard tools from calculus and complex analysis. It is used to deal with KAM theory for finitely smooth systems by Zehnder [32. Also see [9] and [31] and references therein, for example. For ease of notation, we shall replace $\|\cdot\|_{X}$ by $\|\cdot\|$. Now let us apply this lemma to the perturbation $P(\phi)$.

Fix a sequence of fast decreasing numbers $s_{\nu} \downarrow 0, v \geq 0$, and $s_{0} \leq 1 / 2$. For an $X$ valued function $P(\phi)$, construct a sequence of real analytic functions $P^{(v)}(\phi)=P_{s_{v}}(\phi)$ such that the following conclusions hold: 
(1) $P^{(v)}(\phi)$ is real analytic on the complex strip $\mathbb{T}_{s_{v}}^{n}$ of the width $s_{v}$ around $\mathbb{T}^{n}$.

(2) The sequence of functions $P^{(v)}(\phi)$ satisfies the bounds:

$$
\begin{array}{r}
\sup _{\phi \in \mathbb{T}^{n}}\left\|P^{(v)}(\phi)-P(\phi)\right\| \leq C\|P\|_{C^{\ell}} s_{v}^{\ell}, \\
\sup _{\phi \in \mathbb{T}_{s_{v+1}^{n}}^{n}}\left\|P^{(v+1)}(\phi)-P^{(v)}(\phi)\right\| \leq C\|P\|_{C^{\ell}} s_{v}^{\ell},
\end{array}
$$

where $C$ denotes (different) constants depending only on $n$ and $\ell$.

(3) The first approximate $P^{(0)}$ is "small" with the perturbation $P$. Precisely speaking, for arbitrary $\phi \in \mathbb{T}_{s_{0}}^{n}$, we have

$$
\begin{aligned}
\left\|P^{(0)}(\phi)\right\| & \leq\left\|P^{(0)}(\phi)-\sum_{|\alpha| \leq \ell} \frac{\partial^{\alpha} P(\operatorname{Re} \phi)}{\alpha !}(\mathbf{i} \operatorname{Im} \phi)^{\alpha}\right\|+\left\|\sum_{|\alpha| \leq \ell} \frac{\partial^{\alpha} P(\operatorname{Re} \phi)}{\alpha !}(\mathbf{i} \operatorname{Im} \phi)^{\alpha}\right\| \\
& \leq C\left(\|P\|_{C^{\ell}} s_{0}^{\ell}+\sum_{0 \leq m \leq \ell}\|P\|_{C^{m}} s_{0}^{m}\right) \leq C\|P\|_{C^{\ell}} \sum_{m=0}^{\ell} s_{0}^{m} \\
& \leq C\|P\|_{C^{\ell}} \sum_{m=0}^{\infty} s_{0}^{m} \leq C\|P\|_{C^{\ell}},
\end{aligned}
$$

where constant $C$ is independent of $s_{0}$, and the last inequality holds true due to the hypothesis that $s_{0} \leq 1 / 2$.

(4) From the first inequality (3.1), we have the equality below. For arbitrary $\phi \in \mathbb{T}^{n}$,

$$
P(\phi)=P^{(0)}(\phi)+\sum_{v=0}^{+\infty}\left(P^{(v+1)}(\phi)-P^{(v)}(\phi)\right) .
$$

Now take a sequence of real numbers $\left\{s_{v} \geq 0\right\}_{v=0}^{\infty}$ with $s_{v}>s_{v+1}$ goes fast to zero. Let $R^{p, q}(\theta)=P(\theta)$ for $p, q \in\{z, \bar{z}\}$. Then by 3.2 we can write, for $p, q \in\{z, \bar{z}\}$,

$$
R^{p, q}(\theta)=R_{0}^{p, q}(\theta)+\sum_{l=1}^{\infty} R_{l}^{p, q}(\theta),
$$

where $R_{0}^{p, q}(\theta)$ is analytic in $\mathbb{T}_{s_{0}}^{n}$ with

$$
\sup _{\theta \in \mathbb{T}_{s_{0}}^{n}}\left\|R_{0}^{p, q}(\theta)\right\|_{h_{N} \rightarrow h_{N}} \leq C
$$

and $R_{l}^{p, q}(\theta)(l \geq 1)$ is analytic in $\mathbb{T}_{s_{l}}^{n}$ with

$$
\sup _{\theta \in \mathbb{T}_{s_{l}}^{n}}\left\|J R_{l}^{p, q}(\theta) J\right\|_{h_{N} \rightarrow h_{N}} \leq C s_{l-1}^{N}
$$




\section{Iterative parameters of domains}

Let

- $\varepsilon_{0}=\varepsilon, \varepsilon_{\nu}=\varepsilon^{(4 / 3)^{\nu}}, \nu=0,1,2, \ldots$, which measures the size of perturbation at $\nu$-th step.

- $s_{\nu}=\varepsilon_{\nu+1}^{1 / N}, \nu=0,1,2, \ldots$, which measures the strip-width of the analytic domain $\mathbb{T}_{s_{\nu}}^{n}, \mathbb{T}_{s_{\nu}}^{n}=\left\{\theta \in \mathbb{C}^{n} / 2 \pi \mathbb{Z}^{n}:|\operatorname{Im} \theta| \leq s_{\nu}\right\}$

- $C(\nu)$ is a constant which may be different in different places, and it is of the form

$$
C(\nu)=C_{1} 2^{C_{2} \nu}
$$

where $C_{1}, C_{2}$ are constants.

- $K_{\nu}=100 s_{\nu}^{-1} 2^{\nu}|\log \varepsilon|$.

- $\gamma_{\nu}=\gamma / 2^{\nu}, 0<\gamma \ll 1$.

- a family of subsets $\Pi_{\nu} \subset[1,2]$ with $[1,2] \supset \Pi_{0} \supset \cdots \supset \Pi_{\nu} \supset \cdots$, and

$$
\operatorname{mes} \Pi_{\nu} \geq \operatorname{mes} \Pi_{\nu-1}-C \gamma_{\nu-1}^{1 / 3} \text {. }
$$

- For an operator-value (or a vector-value) function $B(\theta, \tau)$, whose domain is $(\theta, \tau) \in$ $\mathbb{T}_{s_{\nu}}^{n} \times \Pi_{\nu}$, set

$$
\|B\|_{\mathbb{T}_{s_{\nu}}^{n} \times \Pi_{\nu}}=\sup _{(\theta, \tau) \in \mathbb{T}_{s_{\nu}}^{n} \times \Pi_{\nu}}\|B(\theta, \tau)\|_{h_{N} \rightarrow h_{N}}
$$

where $\|\cdot\|_{h_{N} \rightarrow h_{N}}$ is the operator norm, and set

$$
\|B\|_{\mathbb{T}_{s_{\nu}}^{n} \times \Pi_{\nu}}^{\mathscr{L}}=\sup _{(\theta, \tau) \in \mathbb{T}_{s_{\nu}}^{n} \times \Pi_{\nu}}\left\|\partial_{\tau} B(\theta, \tau)\right\|_{h_{N} \rightarrow h_{N}} .
$$

\section{Iterative lemma}

In the following, for a function $f(\omega)$, denote by $\partial_{\omega}$ the derivative of $f(\omega)$ with respect to $\omega$ in Whitney's sense.

Lemma 5.1. For $p, q \in\{z, \bar{z}\}$, let $R_{0,0}^{p, q}=R_{0}^{p, q}, R_{l, 0}^{p, q}=R_{l}^{p, q}$, where $R_{0}^{p, q}, R_{l}^{p, q}$ are defined by (3.3), (3.4) and (3.5). Assume that we have a family of Hamiltonian functions $H_{\nu}$ :

$$
H_{\nu}=\sum_{j=1}^{\infty} \lambda_{j}^{(\nu)} z_{j} \bar{z}_{j}+\sum_{l \geq \nu}^{\infty} \varepsilon_{l}\left(\left\langle R_{l, \nu}^{z z} z, z\right\rangle+\left\langle R_{l, \nu}^{z \bar{z}} z, \bar{z}\right\rangle+\left\langle R_{l, \nu}^{\overline{z z}} \bar{z}, \bar{z}\right\rangle\right), \quad \nu=0,1, \ldots, m
$$

where $R_{l, \nu}^{z z}, R_{l, \nu}^{z \bar{z}}, R_{l, \nu}^{\overline{z z}}$ are operator-valued functions defined on the domain $\mathbb{T}_{s_{\nu}}^{n} \times \Pi_{\nu}$, and $\theta=\omega t$. 
$(\mathrm{A} 1)_{\nu}$

$$
\lambda_{j}^{(0)}=\sqrt{\lambda_{j}}=j, \quad \lambda_{j}^{(\nu)}=\sqrt{\lambda_{j}}+\sum_{i=0}^{\nu-1} \varepsilon_{i} \mu_{j}^{(i)}, \quad \nu \geq 1
$$

and $\mu_{j}^{(i)}=\mu_{j}^{(i)}(\tau): \Pi_{i} \rightarrow \mathbb{R}$ with

$$
\begin{aligned}
& \left|\mu_{j}^{(i)}\right|_{\Pi_{i}}:=\sup _{\tau \in \Pi_{i}}\left|\mu_{j}^{(i)}(\tau)\right| \leq C(i) / j, \quad 0 \leq i \leq \nu-1, \\
& \left|\mu_{j}^{(i)}\right|_{\Pi_{i}}^{\mathscr{C}}:=\sup _{\tau \in \Pi_{i}}\left|\partial_{\tau} \mu_{j}^{(i)}(\tau)\right| \leq C(i) / j, \quad 0 \leq i \leq \nu-1 .
\end{aligned}
$$

$(\mathrm{A} 2)_{\nu}$ For $p, q \in\{z, \bar{z}\}, R_{l, \nu}^{p, q}=R_{l, \nu}^{p, q}(\theta, \tau)$ is defined in $\mathbb{T}_{s_{l}}^{n} \times \Pi_{\nu}$ with $l \geq \nu$, and is analytic in $\theta$ for fixed $\tau \in \Pi_{\nu}$, and

$$
\begin{aligned}
\left\|J R_{l, \nu}^{p, q} J\right\|_{\mathbb{T}_{s_{l}}^{n} \times \Pi_{\nu}} & \leq C(\nu), \\
\left\|J R_{l, \nu}^{p, q} J\right\|_{\mathbb{T}_{s_{l}}^{n} \times \Pi_{\nu}}^{\mathscr{L}} & \leq C(\nu) .
\end{aligned}
$$

Then there exists a compact set $\Pi_{m+1} \subset \Pi_{m}$ with

$$
\operatorname{mes} \Pi_{m+1} \geq \operatorname{mes} \Pi_{m}-C \gamma_{m}^{1 / 3}
$$

and symplectic coordinate changes

$$
\begin{gathered}
\Psi_{m}: \mathbb{T}_{s_{m+1}}^{n} \times \Pi_{m+1} \rightarrow \mathbb{T}_{s_{m}}^{n} \times \Pi_{m}, \\
\left\|\Psi_{m}-\mathrm{id}\right\|_{h_{N} \rightarrow h_{N}} \leq \varepsilon^{1 / 2}, \quad(\theta, \tau) \in \mathbb{T}_{s_{m+1}}^{n} \times \Pi_{m+1}
\end{gathered}
$$

such that the Hamiltonian function $H_{m}$ is changed into

$$
\begin{aligned}
H_{m+1} & \triangleq H_{m} \circ \Psi_{m} \\
& =\sum_{j=1}^{\infty} \lambda_{j}^{(m+1)} z_{j} \bar{z}_{j}+\sum_{l \geq m+1}^{\infty} \varepsilon_{l}\left[\left\langle R_{l, m+1}^{z z} z, z\right\rangle+\left\langle R_{l, m+1}^{z \bar{z}} z, \bar{z}\right\rangle+\left\langle R_{l, m+1}^{\bar{z}} \bar{z}, \bar{z}\right\rangle\right],
\end{aligned}
$$

which is defined on the domain $\mathbb{T}_{s_{m+1}}^{n} \times \Pi_{m+1}$, and $\lambda_{j}^{(m+1)}$ 's satisfy Assumption (A1) $)_{m+1}$ and $R_{l, m+1}^{p, q}(p, q \in\{z, \bar{z}\})$ satisfy Assumption $(\mathrm{A} 2)_{m+1}$.

\section{Derivation of homological equations}

Our end is to find a symplectic transformation $\Psi_{\nu}$ such that the terms $R_{l, v}^{z z}, R_{l, v}^{z \bar{z}}, R_{l, v}^{\bar{z} z}$ (with $l=v$ ) disappear. To this end, let $F$ be a linear Hamiltonian of the form

$$
F=\left\langle F^{z z}(\theta, \tau) z, z\right\rangle+\left\langle F^{z \bar{z}}(\theta, \tau) z, \bar{z}\right\rangle+\left\langle F^{\overline{z z}}(\theta, \tau) \bar{z}, \bar{z}\right\rangle,
$$


where $\theta=\omega t,\left(F^{z z}(\theta, \tau)\right)^{T}=F^{z z}(\theta, \tau),\left(F^{z \bar{z}}(\theta, \tau)\right)^{T}=F^{z \bar{z}}(\theta, \tau),\left(F^{\overline{z z}}(\theta, \tau)\right)^{T}=F^{\overline{z z}}(\theta, \tau)$. Moreover, let

$$
\Psi=\Psi_{m}=\left.X_{\varepsilon_{m} F}^{t}\right|_{t=1},
$$

where $X_{\varepsilon_{m} F}^{t}$ is the flow of the Hamiltonian, $X_{\varepsilon_{m} F}$ is the vector field of the Hamiltonian $\varepsilon_{m} F$ with the symplectic structure $\mathbf{i} d z \wedge d \bar{z}$. Let

$$
H_{m+1}=H_{m} \circ \Psi_{m}
$$

By (5.1), we write

$$
H_{m}=N_{m}+R_{m}
$$

with

$$
\begin{gathered}
N_{m}=\sum_{j=1}^{\infty} \lambda_{j}^{(m)} z_{j} \bar{z}_{j}, \\
R_{m}=\sum_{l=m}^{\infty} \varepsilon_{l} R_{l m}, \\
R_{l m}=\left\langle R_{l, m}^{z z}(\theta) z, z\right\rangle+\left\langle R_{l, m}^{z \bar{z}}(\theta) z, \bar{z}\right\rangle+\left\langle R_{l, m}^{\overline{z z}}(\theta) \bar{z}, \bar{z}\right\rangle,
\end{gathered}
$$

where $\left(R_{l, m}^{z z}(\theta)\right)^{T}=R_{l, m}^{z z}(\theta),\left(R_{l, m}^{z \bar{z}}(\theta)\right)^{T}=R_{l, m}^{z \bar{z}}(\theta),\left(R_{l, m}^{\overline{z z}}(\theta)\right)^{T}=R_{l, m}^{\overline{z z}}(\theta)$. Since the Hamiltonian $H_{m}=H_{m}(\omega t, z, \bar{z})$ depends on time $t$, we introduce a fictitious action $I=$ constant, and let $\theta=\omega t$ be angle variable. Then the non-autonomous $H_{m}(\omega t, z, \bar{z})$ can be written as

$$
\omega I+H_{m}(\theta, z, \bar{z})
$$

with symplectic structure $d I \wedge d \theta+\mathbf{i} d z \wedge d \bar{z}$. By combination of 6.1)-6.7) and Taylor formula, we have

$$
\begin{aligned}
H_{m+1}= & H_{m} \circ X_{\varepsilon_{m} F}^{1} \\
= & N_{m}+\varepsilon_{m}\left\{N_{m}, F\right\}+\varepsilon_{m}^{2} \int_{0}^{1}(1-\tau)\left\{\left\{N_{m}, F\right\}, F\right\} \circ X_{\varepsilon_{m} F}^{\tau} d \tau+\varepsilon_{m} \omega \cdot \partial_{\theta} F \\
& +\varepsilon_{m} R_{m m}+\left(\sum_{l=m+1}^{\infty} \varepsilon_{l} R_{l m}\right) \circ X_{\varepsilon_{m} F}^{1}+\varepsilon_{m}^{2} \int_{0}^{1}\left\{R_{m m}, F\right\} \circ X_{\varepsilon_{m} F}^{\tau} d \tau,
\end{aligned}
$$

where $\{\cdot, \cdot\}$ is the Poisson bracket with respect to $\mathbf{i} d z \wedge d \bar{z}$, that is

$$
\{H(z, \bar{z}), F(z, \bar{z})\}=\mathbf{i}\left(\frac{\partial H}{\partial z} \cdot \frac{\partial F}{\partial \bar{z}}-\frac{\partial H}{\partial \bar{z}} \cdot \frac{\partial F}{\partial z}\right)
$$


Let $\Gamma_{K_{m}}$ be a truncation operator. For any

$$
f(\theta)=\sum_{k \in \mathbb{Z}^{n}} \widehat{f}(k) e^{\mathbf{i}\langle k, \theta\rangle}, \quad \theta \in \mathbb{T}^{n},
$$

define, for given $K_{m}>0$,

$$
\begin{aligned}
\Gamma_{K_{m}} f(\theta) & =\left(\Gamma_{K_{m}} f\right)(\theta) \triangleq \sum_{|k| \leq K_{m}} \widehat{f}(k) e^{\mathbf{i}\langle k, \theta\rangle} \\
\left(1-\Gamma_{K_{m}}\right) f(\theta) & =\left(\left(1-\Gamma_{K_{m}}\right) f\right)(\theta) \triangleq \sum_{|k|>K_{m}} \widehat{f}(k) e^{\mathbf{i}\langle k, \theta\rangle} .
\end{aligned}
$$

Then

$$
f(\theta)=\Gamma_{K_{m}} f(\theta)+\left(1-\Gamma_{K_{m}}\right) f(\theta) .
$$

Let

$$
\omega \cdot \partial_{\theta} F+\left\{N_{m}, F\right\}+\Gamma_{K_{m}} R_{m m}=\left\langle\left[R_{m m}^{z \bar{z}}\right] z, \bar{z}\right\rangle,
$$

where

$$
\left[R_{m m}^{z \bar{z}}\right]:=\operatorname{diag}\left(\widehat{R}_{m m j j}^{z \bar{z}}(0): j=1,2, \ldots\right),
$$

and $R_{m m i j}^{z \bar{z}}(\theta)$ is the matrix element of $R_{m, m}^{z \bar{z}}(\theta)$ and $\widehat{R}_{m m i j}^{z \bar{z}}(k)$ is the $k$-Fourier coefficient of $R_{m m i j}^{z \bar{z}}(\theta)$. Then

$$
H_{m+1}=N_{m+1}+C_{m+1} R_{m+1}
$$

where

$$
\begin{aligned}
& N_{m+1}= N_{m}+\varepsilon_{m}\left\langle\left[R_{m m}^{z \bar{z}}\right] z, \bar{z}\right\rangle=\sum_{j=1}^{\infty} \lambda_{j}^{(m+1)} z_{j} \bar{z}_{j} \\
& \lambda_{j}^{(m+1)}=\lambda_{j}^{(m)}+\varepsilon_{m} \widehat{R}_{m m j j}^{z \bar{z}}(0)=\sqrt{\lambda_{j}}+\sum_{l=1}^{m} \varepsilon_{l} \mu_{j}^{(l)}, \quad \mu_{j}^{(m)}:=\widehat{R}_{m m j j}^{z \bar{z}}(0) \\
& C_{m+1} R_{m+1}= \\
& \varepsilon_{m}\left(1-\Gamma_{K_{m}}\right) R_{m m} \\
&+\varepsilon_{m}^{2} \int_{0}^{1}(1-\tau)\left\{\left\{N_{m}, F\right\}, F\right\} \circ X_{\varepsilon_{m} F}^{\tau} d \tau \\
&+\varepsilon_{m}^{2} \int_{0}^{1}\left\{R_{m m}, F\right\} \circ X_{\varepsilon_{m} F}^{\tau} d \tau \\
&+\left(\sum_{l=m+1}^{\infty} \varepsilon_{l} R_{l m}\right) \circ X_{\varepsilon_{m} F}^{1}
\end{aligned}
$$


The equation (6.8) is called homological equation. Developing the Poisson bracket $\left\{N_{m}, F\right\}$ and comparing the coefficients of $z_{i} z_{j}, z_{i} \bar{z}_{j}, \bar{z}_{i} \bar{z}_{j}(i, j=1,2, \ldots)$, we get

$$
\begin{aligned}
& \omega \cdot \partial_{\theta} F^{z z}(\theta, \tau)+\mathbf{i}\left(\Lambda^{(m)} F^{z z}(\theta, \tau)+F^{z z}(\theta, \tau) \Lambda^{(m)}\right)=\Gamma_{K_{m}} R_{m m}^{z z}(\theta), \\
& \omega \cdot \partial_{\theta} F^{\overline{z z}}(\theta, \tau)-\mathbf{i}\left(\Lambda^{(m)} F^{\overline{z z}}(\theta, \tau)+F^{\overline{z z}}(\theta, \tau) \Lambda^{(m)}\right)=\Gamma_{K_{m}} R_{m m}^{\overline{z z}}(\theta), \\
& \omega \cdot \partial_{\theta} F^{z \bar{z}}(\theta, \tau)+\mathbf{i}\left(F^{z \bar{z}}(\theta, \tau) \Lambda^{(m)}-\Lambda^{(m)} F^{z \bar{z}}(\theta, \tau)\right)=\Gamma_{K_{m}} R_{m m}^{z \bar{z}}(\theta)-\left[R_{m m}\right],
\end{aligned}
$$

where

$$
\Lambda^{(m)}=\operatorname{diag}\left(\lambda_{j}^{(m)}: j=1,2, \ldots\right)
$$

and we assume

$$
\Gamma_{K_{m}} F^{z z}(\theta, \tau)=F^{z z}(\theta, \tau), \quad \Gamma_{K_{m}} F^{z \bar{z}}(\theta, \tau)=F^{z \bar{z}}(\theta, \tau), \quad \Gamma_{K_{m}} F^{\overline{z z}}(\theta, \tau)=F^{\overline{z z}}(\theta, \tau) .
$$

Here $F_{i j}^{z z}(\theta), F_{i j}^{z \bar{z}}(\theta), F_{i j}^{\overline{z z}}(\theta)$ are the matrix elements of $F^{z z}(\theta, \tau), F^{z \bar{z}}(\theta, \tau), F^{\overline{z z}}(\theta, \tau)$, respectively. Then 6.14 - 6.16) can be rewritten as

$$
\begin{aligned}
\omega \cdot \partial_{\theta} F_{i j}^{z z}(\theta)+\mathbf{i}\left(\lambda_{i}^{(m)}+\lambda_{j}^{(m)}\right) F_{i j}^{z z}(\theta) & =\Gamma_{K_{m}} R_{m m i j}^{z z}(\theta), \\
\omega \cdot \partial_{\theta} F_{i j}^{\overline{z z}}(\theta)-\mathbf{i}\left(\lambda_{i}^{(m)}+\lambda_{j}^{(m)}\right) F_{i j}^{\overline{z z}}(\theta) & =\Gamma_{K_{m}} R_{m m i j}^{\overline{z z}}(\theta), \\
\omega \cdot \partial_{\theta} F_{i j}^{z \bar{z}}(\theta)-\mathbf{i}\left(\lambda_{i}^{(m)}-\lambda_{j}^{(m)}\right) F_{i j}^{z \bar{z}}(\theta) & =\Gamma_{K_{m}} R_{m m i j}^{z \bar{z}}(\theta), \quad i \neq j, \\
\omega \cdot \partial_{\theta} F_{i i}^{z \bar{z}}(\theta) & =\Gamma_{K_{m}} R_{m m i i}^{z \bar{z}}(\theta)-\widehat{R}_{m m i i}(0),
\end{aligned}
$$

where $i, j=1,2, \ldots$

\section{Solutions of the homological equations}

Lemma 7.1. There exists a compact subset $\Pi_{m+1}^{+-} \subset \Pi_{m}$ with

$$
\operatorname{mes}\left(\Pi_{m+1}^{+-}\right) \geq \operatorname{mes} \Pi_{m}-C \gamma_{m}^{1 / 3}
$$

such that for any $\tau \in \Pi_{m+1}^{+-}$(Recall $\left.\omega=\tau \omega_{0}\right)$, the equation 6.19 has a unique solution $F^{z \bar{z}}(\theta, \tau)$, which is defined on the domain $\mathbb{T}_{s_{m+1}}^{n} \times \Pi_{m+1}^{+-}$, with

$$
\begin{aligned}
& \left\|J F^{z \bar{z}}(\theta, \tau) J\right\|_{\mathbb{T}_{s_{m+1}}^{n} \times \Pi_{m+1}^{+-}} \leq C(m+1) \varepsilon_{m}^{-6(n+1) / N}, \\
& \left\|J F^{z \bar{z}}(\theta, \tau) J\right\|_{\mathbb{T}_{s_{m+1}}^{n} \times \Pi_{m+1}^{+-}}^{\mathscr{L}} \leq C(m+1) \varepsilon_{m}^{-12(n+1) / N} .
\end{aligned}
$$

Proof. By passing to Fourier coefficients, we can rewrite (6.19) as

$$
\left(-\langle k, \omega\rangle+\lambda_{i}^{(m)}-\lambda_{j}^{(m)}\right) \widehat{F}_{i j}^{z \bar{z}}(k)=\mathbf{i} \widehat{R}_{m m i j}^{z \bar{z}}(k),
$$

where $i, j=1,2, \ldots, k \in \mathbb{Z}^{n}$ with $|k| \leq K_{m}$. Recall $\omega=\tau \omega_{0}$. 
Let

$$
A_{k}=|k|^{2 n+3}+8
$$

and let

$$
Q_{k i j}^{(m)} \triangleq\left\{\tau \in \Pi_{m}||-\left\langle k, \omega_{0}\right\rangle \tau+\lambda_{i}^{(m)}-\lambda_{j}^{(m)} \mid<\frac{(|i-j|+1) \gamma_{m}}{A_{k}}\right\}
$$

where $i, j=1,2, \ldots, k \in \mathbb{Z}^{n}$ with $|k| \leq K_{m}$, and $k \neq 0$ when $i=j$. Let

$$
\Pi_{m+1}^{+-}=\Pi_{m} \backslash \bigcup_{|k| \leq K_{m}} \bigcup_{i=1}^{\infty} \bigcup_{j=1}^{\infty} Q_{k i j}^{(m)}
$$

Then for any $\tau \in \Pi_{m+1}^{+-}$, we have

$$
\left|-\langle k, \omega\rangle+\lambda_{i}^{(m)}-\lambda_{j}^{(m)}\right| \geq \frac{(|i-j|+1) \gamma_{m}}{A_{k}} .
$$

Recall that $R_{m m}^{z \bar{z}}(\theta)$ is analytic in the domain $\mathbb{T}_{s_{m}}^{n}$ for any $\tau \in \Pi_{m}$,

$$
\left|\widehat{R}_{m m i j}^{z \bar{z}}(k)\right| \leq \frac{C(m)}{\sqrt{i j}} e^{-s_{m}|k|}
$$

It follows

$$
\begin{aligned}
\left|\widehat{F}_{i j}^{z \bar{z}}(k)\right| & =\left|\frac{\widehat{R}_{m m i j}^{z \bar{z}}(k)}{-\langle k, \omega\rangle+\lambda_{i}^{(m)}-\lambda_{j}^{(m)}}\right| \leq \frac{A_{k}}{\gamma_{m}(|i-j|+1)} \cdot\left|\widehat{R}_{m m i j}^{z \bar{z}}(k)\right| \\
& \leq \frac{\left(|k|^{2 n+3}+8\right)}{\gamma_{m}(|i-j|+1)} \cdot \frac{C(m)}{\sqrt{i j}} e^{-s_{m}|k|} .
\end{aligned}
$$

Now we need the following lemmas:

Lemma 7.2. 6] For $0<\delta<1, \nu>1$, one has

$$
\sum_{k \in \mathbb{Z}^{n}} e^{-2|k| \delta}|k|^{\nu}<\left(\frac{\nu}{e}\right)^{\nu} \frac{(1+e)^{n}}{\delta^{\nu+n}} .
$$

Lemma 7.3. 25. If $A=\left(A_{i j}\right)$ is a bounded linear operator on $\ell^{2}$, then also $B=\left(B_{i j}\right)$ with

$$
B_{i j}=\frac{\left|A_{i j}\right|}{|i-j|}, \quad i \neq j
$$

and $B_{i i}=0$ is a bounded linear operator on $\ell^{2}$, and $\|B\| \leq\left(\frac{\pi}{\sqrt{3}}\right)\|A\|$, where $\|\cdot\|$ is $\ell^{2} \rightarrow \ell^{2}$ operator norm.

Remark 7.4. Lemma 7.3 holds true for the weight norm $\|\cdot\|_{N}$. 
Therefore, by (7.5), we have

$$
\begin{aligned}
& \sup _{\theta \in \mathbb{T}_{s_{m}^{\prime}}^{n} \times \Pi_{m+1}}\left(\sqrt{i}\left|F_{i j}^{z \bar{z}}(\theta, \tau)\right| \sqrt{j}\right) \\
\leq & \left(\sum_{|k| \leq K_{m}}\left(|k|^{2 n+3}+8\right) e^{-\left(s_{m}-s_{m}^{\prime}\right)|k|}\right) \cdot \frac{C(m)}{\gamma_{m}(|i-j|+1)} \\
\leq & \left.C\left(\frac{2 n+3}{e}\right)^{2 n+3}(1+e)^{n}\left(\frac{2}{s_{m}-s_{m}^{\prime}}\right)^{3 n+3} \cdot \frac{C(m)}{\gamma_{m}(|i-j|+1)} \quad \text { by Lemma } 7.2\right) \\
\leq & C \cdot \frac{1}{\left(s_{m}-s_{m}^{\prime}\right)^{3(n+1)}} \cdot \frac{\gamma_{m}(|i-j|+1)}{\gamma_{m}} \\
\leq & C \cdot \varepsilon_{m}^{-6(n+1) / N} \cdot \frac{C(m)}{\gamma_{m}(|i-j|+1)},
\end{aligned}
$$

where $C$ is a constant depending on $n, s_{m}^{\prime}=s_{m}-\left(s_{m}-s_{m+1}\right) / 4$.

By Lemma 7.3 and Remark 7.4, we have

$$
\left\|J F^{z \bar{z}}(\theta, \tau) J\right\|_{\mathbb{T}_{s_{m}^{\prime}}^{n} \times \Pi_{m+1}^{+-}} \leq C \cdot C(m) \gamma_{m}^{-1} \varepsilon_{m}^{-6(n+1) / N} \leq C(m+1) \varepsilon_{m}^{-6(n+1) / N}
$$

It follows from $s_{m}^{\prime}>s_{m+1}$ that

$$
\left\|J F^{z \bar{z}}(\theta, \tau) J\right\|_{\mathbb{T}_{s_{m+1}}^{n} \times \Pi_{m+1}^{+-}} \leq\left\|J F^{z \bar{z}}(\theta, \tau) J\right\|_{\mathbb{T}_{s_{m}^{\prime}}^{n} \times \Pi_{m+1}^{+-}} \leq C(m+1) \varepsilon_{m}^{-6(n+1) / N}
$$

Applying $\partial_{\tau}$ to both sides of $(7.2)$, we have

$$
\left(-\langle k, \omega\rangle+\lambda_{i}^{(m)}-\lambda_{j}^{(m)}\right) \partial_{\tau} \widehat{F}_{i j}^{z \bar{z}}(k)=\mathbf{i} \partial_{\tau} \widehat{R}_{m m i j}^{z \bar{z}}(k)+(*),
$$

where

$$
(*)=-\left(-\left\langle k, \omega_{0}\right\rangle+\partial_{\tau}\left(\lambda_{i}^{(m)}-\lambda_{j}^{(m)}\right)\right) \widehat{F}_{i j}^{z \bar{z}}(k) .
$$

Recalling $|k| \leq K_{m}=100 s_{m}^{-1} 2^{m}|\log \varepsilon|$, and using (5.2) and 5.3 with $\nu=m$, and using (7.6), we have, on $\tau \in \Pi_{m+1}$,

$$
\sqrt{i}|(*)| \sqrt{j} \leq C(m) K_{m}\left|\widehat{F}_{i j}^{z \bar{z}}(k)\right| \text {. }
$$

According to 5.6 ,

$$
\left|\sqrt{i} \partial_{\tau} \widehat{R}_{m m i j}^{z \bar{z}}(k) \sqrt{j}\right| \leq C(m+1) e^{-s_{m}^{*}|k|}
$$

By (7.4), 7.7), 7.8 and (7.9), we have

$$
\left|\sqrt{i} \partial_{\tau} \widehat{F}_{i j}^{z \bar{z}}(k) \sqrt{j}\right| \leq \frac{A_{k}}{\gamma_{m}(|i-j|+1)} \cdot C \cdot C(m+1) K_{m} \gamma_{m}^{-1} \varepsilon_{m}^{-6(n+1) / N} e^{-s_{m}^{\prime}|k|} \quad \text { for } i \neq j .
$$


Note that $s_{m}>s_{m}^{\prime}>s_{m+1}$. Again using Lemmas 7.2 and 7.3 , we have

$$
\begin{aligned}
\left\|J F^{z \bar{z}}(\theta, \tau) J\right\|_{\mathbb{T}_{s_{m+1}}^{\mathscr{L}} \times \Pi_{m+1}^{+-}} & =\left\|J \partial_{\tau} F^{z \bar{z}}(\theta, \tau) J\right\|_{\mathbb{T}_{s_{m+1}} \times \Pi_{m+1}^{+-}} \\
& \leq C^{2} \cdot C(m+1) K_{m} \gamma_{m}^{-1} \varepsilon_{m}^{-12(n+1) / N} \leq C(m+1) \varepsilon_{m}^{-12(n+1) / N} .
\end{aligned}
$$

The proof of the measure estimate (7.1) will be postponed to Section 10. This completes the proof of Lemma 7.1 .

Lemma 7.5. There exists a compact subset $\Pi_{m+1}^{++} \subset \Pi_{m}$ with

$$
\operatorname{mes}\left(\Pi_{m+1}^{++}\right) \geq \operatorname{mes} \Pi_{m}-C \gamma_{m}^{1 / 3}
$$

such that for any $\tau \in \Pi_{m+1}^{++}$(Recall $\left.\omega=\tau \omega_{0}\right)$, the equation 6.17) has a unique solution $F^{z z}(\theta)$, which is defined on the domain $\mathbb{T}_{s_{m+1}}^{n} \times \Pi_{m+1}^{++}$, with

$$
\begin{aligned}
& \left\|J F^{z z}(\theta, \tau) J\right\|_{\mathbb{T}_{s_{m+1}}^{n} \times \Pi_{m+1}^{++}} \leq C(m+1) \varepsilon_{m}^{-6(n+1) / N}, \\
& \left\|J F^{z z}(\theta, \tau) J\right\|_{\mathbb{T}_{s_{m+1}}^{n} \times \Pi_{m+1}^{++}}^{\mathscr{L}} \leq C(m+1) \varepsilon_{m}^{-12(n+1) / N} .
\end{aligned}
$$

Lemma 7.6. There exists a compact subset $\Pi_{m+1}^{--} \subset \Pi_{m}$ with

$$
\operatorname{mes}\left(\Pi_{m+1}^{--}\right) \geq \operatorname{mes} \Pi_{m}-C \gamma_{m}^{1 / 3}
$$

such that for any $\tau \in \Pi_{m+1}^{--}$(Recall $\left.\omega=\tau \omega_{0}\right)$, the equation (6.18) has a unique solution $F^{\overline{z z}}(\theta)$, which is defined on the domain $\mathbb{T}_{s_{m+1}}^{n} \times \Pi_{m+1}^{--}$, with

$$
\begin{gathered}
\left\|J F^{\overline{z z}}(\theta, \tau) J\right\|_{\mathbb{T}_{s_{m+1}}^{n} \times \Pi_{m+1}^{--}} \leq C(m+1) \varepsilon_{m}^{-6(n+1) / N}, \\
\left\|J F^{\overline{z z}}(\theta, \tau) J\right\|_{\mathbb{T}_{s_{m+1}}^{L^{n}} \times \Pi_{m+1}^{--}} \leq C(m+1) \varepsilon_{m}^{-12(n+1) / N} .
\end{gathered}
$$

The proofs of Lemmas 7.5 and 7.6 are a little bit simpler than that of Lemma 7.1. So we omit them.

Let

$$
\Pi_{m+1}=\Pi_{m+1}^{+-} \cap \Pi_{m+1}^{++} \cap \Pi_{m+1}^{--} .
$$

By (7.1), 7.10) and (7.11), we have

$$
\operatorname{mes} \Pi_{m+1} \geq \operatorname{mes} \Pi_{m}-C \gamma_{m}^{1 / 3} \text {. }
$$

8. Coordinate change $\Psi$ by $\varepsilon_{m} F$

Recall $\Psi=\Psi_{m}=\left.X_{\varepsilon_{m} F}^{t}\right|_{t=1}$, where $X_{\varepsilon_{m} F}^{t}$ is the flow of the Hamiltonian $\varepsilon_{m} F$, vector field $X_{\varepsilon_{m} F}$ with symplectic $\mathbf{i} d z \wedge d \bar{z}$. So

$$
\mathbf{i} \dot{z}=\varepsilon_{m} \frac{\partial F}{\partial \bar{z}}, \quad-\mathbf{i} \dot{\bar{z}}=\varepsilon_{m} \frac{\partial F}{\partial z}, \quad \dot{\theta}=\omega .
$$


More exactly,

$$
\begin{aligned}
& \mathbf{i} \dot{z}=\varepsilon_{m}\left(F^{z \bar{z}}(\theta, \tau) z+2 F^{\overline{z z}}(\theta, \tau) \bar{z}\right), \quad \theta=\omega t, \\
& -\mathbf{i} \dot{\bar{z}}=\varepsilon_{m}\left(2 F^{z z}(\theta, \tau) z+F^{z \bar{z}}(\theta, \tau) \bar{z}\right), \quad \theta=\omega t, \\
& \dot{\theta}=\omega \text {. }
\end{aligned}
$$

Let $u=\left(\frac{z}{z}\right)$,

$$
B_{m}=\left(\begin{array}{cc}
-\mathbf{i} F^{z \bar{z}}(\theta, \tau) & -2 \mathbf{i} F^{\overline{z z}}(\theta, \tau) \\
2 \mathbf{i} F^{z z}(\theta, \tau) & \mathbf{i} F^{z \bar{z}}(\theta, \tau)
\end{array}\right), \quad \theta=\omega t
$$

Then

$$
\frac{d u(t)}{d t}=\varepsilon_{m} B_{m}(\theta) u, \quad \dot{\theta}=\omega .
$$

Let $u(0)=u_{0} \in h_{N} \times h_{N}, \theta(0)=\theta_{0} \in \mathbb{T}_{s_{m+1}}^{n}$ be initial value. Then

$$
u(t)=u_{0}+\int_{0}^{t} \varepsilon_{m} B_{m}\left(\theta_{0}+\omega s\right) u(s) d s, \quad \theta(t)=\theta_{0}+\omega t .
$$

By Lemmas 7.1, 7.5 and 7.6,

$$
\begin{aligned}
&\left\|J B_{m}(\theta) J\right\|_{\mathbb{T}_{s_{m+1}}^{n} \times \Pi_{m+1}} \leq C(m+1) \varepsilon_{m}^{-6(n+1) / N}, \\
&\left\|J B_{m}(\theta) J\right\|_{\mathbb{T}_{s_{m+1}}^{n} \times \Pi_{m+1}}^{\mathscr{L}} \leq C(m+1) \varepsilon_{m}^{-12(n+1) / N} .
\end{aligned}
$$

It follows from 8.2 that

$$
u(t)-u_{0}=\int_{0}^{t} \varepsilon_{m} B_{m}\left(\theta_{0}+\omega s\right) u_{0} d s+\int_{0}^{t} \varepsilon_{m} B_{m}\left(\theta_{0}+\omega s\right)\left(u(s)-u_{0}\right) d s .
$$

Moreover, for $t \in[0,1],\left\|u_{0}\right\|_{N} \leq 1$,

$$
\left\|u(t)-u_{0}\right\|_{N} \leq \varepsilon_{m} C(m+1) \varepsilon_{m}^{-6(n+1) / N}+\int_{0}^{t} \varepsilon_{m}\left\|B_{m}\left(\theta_{0}+\omega s\right)\right\|\left\|u(s)-u_{0}\right\|_{N} d s
$$

where $\|\cdot\|$ is the operator norm from $h_{N} \times h_{N} \rightarrow h_{N} \times h_{N}$.

By Gronwall's inequality,

$$
\left\|u(t)-u_{0}\right\|_{N} \leq C(m+1) \varepsilon_{m}^{1-6(n+1) / N} \cdot \exp \left(\int_{0}^{t} \varepsilon_{m}\left\|B_{m}\left(\theta_{0}+\omega s\right)\right\| d s\right) \leq \varepsilon_{m}^{1 / 2} .
$$

Thus,

$$
\Psi_{m}: \mathbb{T}_{s_{m+1}}^{n} \times \Pi_{m+1} \rightarrow \mathbb{T}_{s_{m}}^{n} \times \Pi_{m}
$$

and

$$
\left\|\Psi_{m}-\mathrm{id}\right\|_{h_{N} \rightarrow h_{N}} \leq \varepsilon_{m}^{1 / 2}
$$


Since 8.1 is linear, so $\Psi_{m}$ is linear coordinate change. According to 8.2 , construct Picard sequence

$$
u_{0}(t)=u_{0}, \quad u_{j+1}(t)=u_{0}+\int_{0}^{t} \varepsilon_{m} B\left(\theta_{0}+\omega s\right) u_{j}(s) d s, \quad j=0,1,2, \ldots
$$

By (8.3), this sequence with $t=1$ goes to

$$
\Psi_{m}\left(u_{0}\right)=u(1)=\left(\mathrm{id}+P_{m}\left(\theta_{0}\right)\right) u_{0},
$$

where id is the identity from $h_{N} \times h_{N} \rightarrow h_{N} \times h_{N}$, and $P_{m}\left(\theta_{0}\right)$ is an operator form $h_{N} \times h_{N} \rightarrow h_{N} \times h_{N}$ for any fixed $\theta_{0} \in \mathbb{T}_{s_{m+1}}^{n}, \tau \in \Pi_{m+1}$, and is analytic in $\theta_{0} \in \mathbb{T}_{s_{m+1}}^{n}$, with

$$
\left\|P_{m}\left(\theta_{0}\right)\right\|_{\mathbb{T}_{s_{m+1}}^{n} \times \Pi_{m+1}} \leq \varepsilon_{m}^{1 / 2} .
$$

Note that 8.1$)$ is a Hamiltonian system. So $P_{m}\left(\theta_{0}\right)$ is a symplectic linear operator from $h_{N} \times h_{N}$ to $h_{N} \times h_{N}$.

\section{Estimates of remainders}

The section is aimed to estimate the remainders:

$$
C_{m+1} R_{m+1}=6.10+\cdots+6.13 .
$$

Case 1: Estimate of 6.10). By (6.7), let

$$
\widetilde{R}_{m m}=\widetilde{R}_{m m}(\theta)=\left(\begin{array}{cc}
R_{m, m}^{z z}(\theta) & \frac{1}{2} R_{m, m}^{z \bar{z}}(\theta) \\
\frac{1}{2} R_{m, m}^{z \bar{z}}(\theta) & R_{m, m}^{\overline{z z}}(\theta)
\end{array}\right)
$$

then

$$
R_{m m}=\left\langle\widetilde{R}_{m m}\left(\begin{array}{l}
z \\
\bar{z}
\end{array}\right),\left(\begin{array}{l}
z \\
\bar{z}
\end{array}\right)\right\rangle
$$

So

$$
\left(1-\Gamma_{K_{m}}\right) R_{m m} \triangleq\left\langle\left(1-\Gamma_{K_{m}}\right) \widetilde{R}_{m m}\left(\begin{array}{c}
z \\
\bar{z}
\end{array}\right),\left(\begin{array}{c}
z \\
\bar{z}
\end{array}\right)\right\rangle .
$$

By the definition of truncation operator $\Gamma_{K_{m}}$,

$$
\left(1-\Gamma_{K_{m}}\right) \widetilde{R}_{m m}=\sum_{|k|>K_{m}} \widehat{\widetilde{R}}_{m m}(k) e^{i\langle k, \theta\rangle}, \quad \theta \in \mathbb{T}_{s_{m}}^{n}, \tau \in \Pi_{m}
$$

Since $\widetilde{R}_{m m}=\widetilde{R}_{m m}(\theta)$ is analytic in $\theta \in \mathbb{T}_{s_{m}}^{n}$,

$$
\sup _{(\theta, \tau) \in \mathbb{T}_{s_{m+1}}^{n} \times \Pi_{m+1}}\left\|J\left(1-\Gamma_{K_{m}}\right) \widetilde{R}_{m m} J\right\|_{h_{N} \rightarrow h_{N}}^{2} \leq \sum_{|k|>K_{m}}\left\|J \widehat{\widetilde{R}}_{m m}(k) J\right\|_{N}^{2} e^{2|k| s_{m+1}}
$$




$$
\begin{aligned}
& \leq\left\|J \widetilde{R}_{m m} J\right\|_{\mathbb{T}_{s m}^{n} \times \Pi_{m}}^{2} \sum_{|k|>K_{m}} e^{-2\left(s_{m}-s_{m+1}\right)|k|} \\
& \left.\leq C^{2}(m) \varepsilon_{m}^{-1} e^{-2 K_{m}\left(s_{m}-s_{m+1}\right)} \quad \text { (by }(5.5)\right) \\
& \leq C^{2}(m) \varepsilon_{m}^{2} .
\end{aligned}
$$

That is,

$$
\left\|J\left(1-\Gamma_{K_{m}}\right) \widetilde{R}_{m m} J\right\|_{\mathbb{T}_{s_{m+1}}^{n} \times \Pi_{m+1}} \leq \varepsilon_{m} C(m)
$$

Thus,

$$
\left\|\varepsilon_{m} J\left(1-\Gamma_{K_{m}}\right) \widetilde{R}_{m m} J\right\|_{\mathbb{T}_{s_{m+1}}^{n} \times \Pi_{m+1}} \leq \varepsilon_{m}^{2} C(m) \leq \varepsilon_{m+1} C(m+1) .
$$

Similarly,

$$
\left\|\varepsilon_{m} J\left(1-\Gamma_{K_{m}}\right) \widetilde{R}_{m m} J\right\|_{\mathbb{T}_{s_{m+1}}^{n}}^{\mathscr{L}} \times \Pi_{m+1} \leq \varepsilon_{m+1} C(m+1) .
$$

Case 2: Estimate of 6.12. Let

$$
S_{m}=\left(\begin{array}{cc}
F^{z z}(\theta, \tau) & \frac{1}{2} F^{z \bar{z}}(\theta, \tau) \\
\frac{1}{2} F^{z \bar{z}}(\theta, \tau) & F^{\overline{z z}}(\theta, \tau)
\end{array}\right), \quad \mathscr{J}=\left(\begin{array}{cc}
0 & -\mathbf{i} \text { id } \\
\mathbf{i} \text { id } & 0
\end{array}\right)
$$

Then we can write

$$
F=\left\langle S_{m}(\theta)\left(\begin{array}{l}
z \\
\bar{z}
\end{array}\right),\left(\begin{array}{l}
z \\
\bar{z}
\end{array}\right)\right\rangle=\left\langle S_{m} u, u\right\rangle, \quad u=\left(\begin{array}{l}
z \\
\bar{z}
\end{array}\right) .
$$

Then

$$
\varepsilon_{m}^{2}\left\{R_{m m}, F\right\}=4 \varepsilon_{m}^{2}\left\langle\widetilde{R}_{m m}(\theta) \mathscr{J} S_{m}(\theta) u, u\right\rangle .
$$

Noting $\mathbb{T}_{s_{m}}^{n} \times \Pi_{m} \supset \mathbb{T}_{s_{m+1}}^{n} \times \Pi_{m+1}$. By (5.6) with $l=m, v=m$,

$$
\begin{aligned}
& \left\|\widetilde{R}_{m m}(\theta)\right\|_{\mathbb{T}_{s_{m+1}}^{n} \times \Pi_{m+1}} \leq\left\|\widetilde{R}_{m m}(\theta)\right\|_{\mathbb{T}_{s_{m}}^{n} \times \Pi_{m}} \leq C(m), \\
& \left\|\widetilde{R}_{m m}(\theta)\right\|_{\mathbb{T}_{s_{m+1}}^{n} \times \Pi_{m+1}}^{\mathscr{L}} \leq C(m) .
\end{aligned}
$$

Let $\widetilde{S}_{m}(\theta)=\mathscr{J} S_{m}(\theta)$. Then by Lemmas 7.1, 7.5 and 7.6, we have

$$
\begin{aligned}
\left\|J \widetilde{S}_{m}(\theta) J\right\|_{\mathbb{T}_{s_{m+1}}^{n} \times \Pi_{m+1}} & \leq C(m+1) \varepsilon_{m}^{-6(n+1) / N} \\
\left\|J \widetilde{S}_{m}(\theta) J\right\|_{\mathbb{T}_{s_{m+1}}^{n}}^{\mathscr{L}} \times \Pi_{m+1} & \leq C(m+1) \varepsilon_{m}^{-12(n+1) / N}
\end{aligned}
$$

and

$$
\begin{aligned}
\left\|\widetilde{R}_{m m} \mathscr{J} S_{m}\right\|_{\mathbb{T}_{s_{m+1}}^{n} \times \Pi_{m+1}} & =\left\|\widetilde{R}_{m m} \widetilde{S}_{m}\right\|_{\mathbb{T}_{s_{m+1}}^{n} \times \Pi_{m+1}} \\
& \leq C(m) C(m+1) \varepsilon_{m}^{-6(n+1) / N}
\end{aligned}
$$


Set

$$
\left[\widetilde{R}_{m m}, \widetilde{S}_{m}\right]=\widetilde{R}_{m m} \widetilde{S}_{m}+\left(\widetilde{R}_{m m} \widetilde{S}_{m}\right)^{T} .
$$

Note that the vector field is linear. So, by Taylor formula, one has

$$
6.12=\varepsilon_{m}^{2}\left\langle\widetilde{R}_{m}^{*}(\theta) u, u\right\rangle
$$

where

$$
\widetilde{R}_{m}^{*}(\theta)=2^{2} \widetilde{R}_{m m} \widetilde{S}_{m}+\sum_{j=2}^{\infty} \frac{2^{j+1} \varepsilon_{m}^{j-1}}{j !} \underbrace{\left[\cdots\left[\widetilde{R}_{m m}, \widetilde{S}_{m}\right], \ldots, \widetilde{S}_{m}\right]}_{(j-1) \text {-fold }} \widetilde{S}_{m} .
$$

By 9.1 and 9.3 ,

$$
\begin{aligned}
\left\|J \widetilde{R}_{m}^{*}(\theta) J\right\|_{\mathbb{T}_{s_{m+1}}^{n} \times \Pi_{m+1}} & \leq \sum_{j=1}^{\infty} \frac{C(m) C(m+1) \varepsilon_{m}^{j-1}\left(\varepsilon_{m}^{-6(n+1) / N}\right)^{j}}{j !} \\
& \leq C(m) C(m+1) \varepsilon_{m}^{-6(n+1) / N} .
\end{aligned}
$$

By $(9.2$ and (9.4),

$$
\left\|J \widetilde{R}_{m}^{*}(\theta) J\right\|_{\mathbb{T}_{s_{m+1}}^{n}}^{\mathscr{L}} \times \Pi_{m+1} \leq C(m) C(m+1) \varepsilon_{m}^{-12(n+1) / N} .
$$

Thus,

$$
\left\|\varepsilon_{m}^{2} J \widetilde{R}_{m}^{*} J\right\|_{\mathbb{T}_{s_{m+1}}^{n} \times \Pi_{m+1}} \leq C(m) C(m+1) \varepsilon_{m}^{2-6(n+1) / N} \leq C(m+1) \varepsilon_{m+1}
$$

and

$$
\left\|\varepsilon_{m}^{2} J \widetilde{R}_{m}^{*} J\right\|_{\mathbb{T}_{s_{m+1}}^{n}}^{\mathscr{L}} \times \Pi_{m+1} \leq C(m) C(m+1) \varepsilon_{m}^{2-12(n+1) / N} \leq C(m+1) \varepsilon_{m+1} .
$$

Case 3: Estimate of 6.11. By 6.8,

$$
\left\{N_{m}, F\right\}=\left\langle\left[R_{m m}^{z \bar{z}}\right] z, \bar{z}\right\rangle-\Gamma_{K_{m}} R_{m m}-\omega \cdot \partial_{\theta} F \triangleq R_{m m}^{*}
$$

Thus,

$$
6.11)=\varepsilon_{m}^{2} \int_{0}^{1}(1-\tau)\left\{R_{m m}^{*}, F\right\} \circ X_{\varepsilon_{m} F}^{\tau} d \tau .
$$

Note $R_{m m}^{*}$ is a quadratic polynomial in $z$ and $\bar{z}$. So we write

$$
R_{m m}^{*}=\left\langle\mathscr{R}_{m}(\theta, \tau) u, u\right\rangle, \quad u=\left(\begin{array}{c}
z \\
\bar{z}
\end{array}\right) .
$$

By (5.3) and (5.4) with $l=\nu=m$, and using (9.3) and (9.4),

$$
\begin{aligned}
&\left\|J \mathscr{R}_{m} J\right\|_{\mathbb{T}_{s_{m}+1}^{n} \times \Pi_{m+1}} \leq C(m) \varepsilon_{m}^{-6(n+1) / N}, \\
&\left\|J \mathscr{R}_{m} J\right\|_{\mathbb{T}_{s_{m}+1}^{n} \times \Pi_{m+1}}^{\mathscr{L}} \leq C(m) \varepsilon_{m}^{-12(n+1) / N},
\end{aligned}
$$


where $\|\cdot\|$ is the operator norm in $h_{N} \times h_{N} \rightarrow h_{N} \times h_{N}$. Recall $F=\left\langle S_{m}(\theta, \tau) u, u\right\rangle$. Set

$$
\left[\mathscr{R}_{m}, \widetilde{S}_{m}\right]=\mathscr{R}_{m} \widetilde{S}_{m}+\left(\mathscr{R}_{m} \widetilde{S}_{m}\right)^{T}
$$

Using Taylor formula to 9.5 , we get

$$
\begin{aligned}
6.11 & =\frac{\varepsilon_{m}^{2}}{2 !}\left\{\left\{R_{m m}^{*}, F\right\}, F\right\}+\cdots+\frac{\varepsilon_{m}^{j}}{j !} \underbrace{\left\{\cdots\left\{R_{m m}^{*}, F\right\}, \ldots, F\right\}}_{j \text {-fold }}+\cdots \\
& =\left\langle(\sum_{j=2}^{\infty} \frac{2^{j+1} \varepsilon_{m}^{j}}{j !} \underbrace{\left[\cdots\left[\mathscr{R}_{m}, \widetilde{S}_{m}\right], \ldots, \widetilde{S}_{m}\right]}_{(j-1) \text {-fold }} \widetilde{S}_{m}) \widetilde{u}, \widetilde{u}\right\rangle \\
& \triangleq\left\langle\mathscr{R}^{* *}(\theta, \tau) u, u\right\rangle .
\end{aligned}
$$

By (9.3), 9.6) and (9.7), we have

$$
\begin{aligned}
& \left\|J \mathscr{R}^{* *}(\theta, \tau) J\right\|_{\mathbb{T}_{s_{m}+1}^{n} \times \Pi_{m+1}} \\
\leq & \sum_{j=2}^{\infty} \frac{2^{j+1}}{j !}\left\|J \mathscr{R}_{m}(\theta, \tau) J\right\|_{\mathbb{T}_{s_{m}}^{n} \times \Pi_{m}}\left(\left\|J \widetilde{S}_{m} J\right\|_{\mathbb{T}_{s_{m+1}}^{n} \times \Pi_{m+1}} \varepsilon_{m}\right)^{j} \\
\leq & \sum_{j=2}^{\infty} \frac{C(m)}{j !}\left(\varepsilon_{m} C(m+1) \varepsilon_{m}^{-6(n+1) / N}\right)^{j} \\
\leq & C(m+1) \varepsilon_{m}^{4 / 3}=C(m+1) \varepsilon_{m+1} .
\end{aligned}
$$

Similarly,

$$
\left\|J \mathscr{R}^{* *} J\right\|_{\mathbb{T}_{s_{m+1}}^{n}}^{\mathscr{L}} \times \Pi_{m+1} \leq C(m+1) \varepsilon_{m+1} .
$$

Case 4: Estimate of 6.13.

$$
6.13)=\sum_{l=m+1}^{\infty} \varepsilon_{l}\left(R_{l m} \circ X_{\varepsilon_{m} F}^{1}\right) .
$$

Write $R_{l m}=\left\langle\widetilde{R}_{l m}(\theta) u, u\right\rangle$. Then, by Taylor formula

$$
R_{l m} \circ X_{\varepsilon_{m} F}^{1}=R_{l m}+\sum_{j=1}^{\infty} \frac{1}{j !}\left\langle\widetilde{R}_{l m j} u, u\right\rangle,
$$

where

$$
\widetilde{R}_{l m j}=2^{j+1} \underbrace{\left[\cdots\left[\widetilde{R}_{l m}, \widetilde{S}_{m}\right], \ldots\right]}_{(j-1) \text {-fold }} \widetilde{S}_{m} \varepsilon_{m}^{j} .
$$

By 5.5, 5.6,

$$
\left\|J \widetilde{R}_{l m} J\right\|_{\mathbb{T}_{s_{l}}^{n} \times \Pi_{m}} \leq C(l), \quad\left\|J \widetilde{R}_{l m} J\right\|_{\mathbb{T}_{s_{l}}^{n} \times \Pi_{m}}^{\mathscr{L}} \leq C(l) .
$$


Combing the last inequalities with $(9.3)$ and $(9.4)$, we have

$$
\begin{aligned}
\left\|J \widetilde{R}_{l m j} J\right\|_{\mathbb{T}_{s_{l}}^{n} \times \Pi_{m+1}} & \leq\left\|J \widetilde{R}_{l m} J\right\|_{\mathbb{T}_{s_{l}}^{n} \times \Pi_{m+1}} \cdot\left(\left\|J \widetilde{S}_{m} J\right\|_{\mathbb{T}_{s_{l}}^{n} \times \Pi_{m+1}} 4 \varepsilon_{m}\right)^{j} \\
& \leq C^{2}(m)\left(\varepsilon_{m} \varepsilon_{m}^{-6(n+1) / N}\right)^{j}
\end{aligned}
$$

where we use $\left\|J^{-1}\right\|_{\mathbb{T}_{s l}^{n} \times \Pi_{m+1}} \leq C$, and

$$
\begin{aligned}
\left\|J \widetilde{R}_{l m j} J\right\|_{\mathbb{T}_{s_{l}}^{n} \times \Pi_{m+1}}^{\mathscr{L}} \leq & \left\|J \widetilde{R}_{l m} J\right\|_{\mathbb{T}_{s_{l}}^{n} \times \Pi_{m+1}}^{\mathscr{L}}\left(\left\|J \widetilde{S}_{m} J\right\|_{\mathbb{T}_{s_{l}}^{n} \times \Pi_{m+1}} 4 \varepsilon_{m}\right)^{j} \\
& +\left\|J \widetilde{R}_{l m} J\right\|_{\mathbb{T}_{s_{l}}^{n} \times \Pi_{m+1}}\left(\left\|J \widetilde{S}_{m} J\right\|_{\mathbb{T}_{s_{l}}^{n} \times \Pi_{m+1}}^{\mathscr{L}} \varepsilon_{m}\right)^{j} \\
\leq & C^{2}(m)\left(\varepsilon_{m} \varepsilon_{m}^{-12(n+1) / N}\right)^{j} .
\end{aligned}
$$

Thus, let

$$
\bar{R}_{l, m+1}:=\widetilde{R}_{l m}+\sum_{j=1}^{\infty} \frac{1}{j !} \widetilde{R}_{l m j}
$$

then

$$
6.13)=\sum_{l=m+1}^{\infty} \varepsilon_{l}\left\langle\bar{R}_{l, m+1} u, u\right\rangle
$$

and

$$
\begin{aligned}
& \left\|J \bar{R}_{l, m+1} J\right\|_{\mathbb{T}_{s_{l}}^{n} \times \Pi_{m+1}} \leq C^{2}(m) \leq C(m+1), \\
& \left\|J \bar{R}_{l, m+1} J\right\|_{\mathbb{T}_{s_{l}}^{n} \times \Pi_{m+1}}^{\mathscr{L}} \leq C^{2}(m) \leq C(m+1) .
\end{aligned}
$$

As a whole, the remainder $R_{m+1}$ can be written as

$$
C_{m+1} R_{m+1}=\sum_{l=m+1}^{\infty} \varepsilon_{l}\left(\left\langle R_{l, \nu}^{z z}(\theta) z, z\right\rangle+\left\langle R_{l, \nu}^{z \bar{z}}(\theta) z, \bar{z}\right\rangle+\left\langle R_{l, \nu}^{\overline{z z}}(\theta) \bar{z}, \bar{z}\right\rangle\right), \quad \nu=m+1,
$$

where, for $p, q \in\{z, \bar{z}\}, R_{l \nu}^{p, q}$ satisfies (5.5) and (5.6) with $\nu=m+1, l \geq m+1$. This shows that Assumption $(\mathrm{A} 2)_{\nu}$ with $\nu=m+1$ holds true.

By (6.9),

$$
\mu_{j}^{(m)}=\widehat{R}_{m m j j}^{z \bar{z}}(0)
$$

In (5.5) and (5.6), taking $p=z, q=\bar{z}$, we have

$$
\begin{aligned}
\left|\mu_{j}^{(m)}\right|_{\Pi_{m}} \leq\left|R_{m m j j}^{z \bar{z}}(\theta, \tau)\right| / j \leq C(m) / j, \\
\left|\mu_{j}^{(m)}\right|_{\Pi_{m}}^{\mathscr{L}} \leq\left|\partial_{\tau} R_{m m j j}^{z \bar{z}}(\theta, \tau)\right| / j \leq C(m) / j .
\end{aligned}
$$

This shows that Assumption $(\mathrm{A} 1)_{\nu}$ with $\nu=m+1$ holds true. 


\section{Estimate of measure}

In this section, $C$ denotes a universal constant, which may be different in different places. Now let us return to 7.3 .

$$
Q_{k i j}^{(m)} \triangleq\left\{\tau \in \Pi_{m}||-\left\langle k, \omega_{0}\right\rangle \tau+\lambda_{i}^{(m)}-\lambda_{j}^{(m)} \mid<\frac{(|i-j|+1) \gamma_{m}}{A_{k}}\right\} .
$$

First let $i=j$, then $k \neq 0$. At this time, (10.1) becomes

$$
Q_{k i i}^{(m)}=\left\{\tau \in \Pi_{m}||\left\langle k, \omega_{0}\right\rangle \tau \mid<\frac{\gamma_{m}}{A_{k}}\right\} .
$$

It follows

$$
\left|\left\langle k, \omega_{0}\right\rangle\right|<\frac{\gamma_{m}}{\left(|k|^{2 n+3}+8\right) \tau} .
$$

Recall $\left|\left\langle k, \omega_{0}\right\rangle\right|>\gamma /|k|^{n+1}$. Then

$$
\operatorname{mes} Q_{k i i}^{(m)}=0
$$

In the following, let $i \neq j$. If $Q_{k i j}^{(m)}=\emptyset$, then mes $Q_{k i j}^{(m)}=0$. So we assume $Q_{k i j}^{(m)} \neq \emptyset$. Then $\exists \tau \in \Pi_{m}$ such that

$$
\left|-\left\langle k, \omega_{0}\right\rangle \tau+\lambda_{i}^{(m)}-\lambda_{j}^{(m)}\right|<\frac{|i-j|+1}{A_{k}} \gamma_{m} .
$$

It follows from (5.2) and (5.3) that

$$
\lambda_{i}^{(m)}-\lambda_{j}^{(m)}=i-j+O\left(\varepsilon_{0} / i\right)+O\left(\varepsilon_{0} / j\right) .
$$

Moreover,

$$
\left|\lambda_{i}^{(m)}-\lambda_{j}^{(m)}\right| \geq \frac{1}{2}|i-j|
$$

By $(10.3)$ and $(10.5)$, one has

$$
\left|\left\langle k, \omega_{0}\right\rangle \tau\right| \geq\left|\lambda_{i}^{(m)}-\lambda_{j}^{(m)}\right|-\frac{|i-j|+1}{A_{k}} \gamma_{m} \geq \frac{1}{2}|i-j|-\frac{|i-j|+1}{A_{k}} \gamma_{m} \geq \frac{1}{4}|i-j| .
$$

Recall $\omega=\omega_{0} \tau$. So

$$
4|\langle k, \omega\rangle| \geq|i-j|
$$

Again by (10.3) and 10.4, we have that, when $\tau \in \Pi_{m}$ such that 10.3 holds true, the following inequality holds true:

$$
\begin{aligned}
|-\langle k, \omega\rangle+i-j| & \leq \frac{|i-j|+1}{A_{k}} \gamma_{m}+\frac{C_{1} \varepsilon_{0}}{i}+\frac{C_{2} \varepsilon_{0}}{j} \\
& \leq \frac{|i-j|+1}{A_{k}} \gamma_{m}+\frac{C_{1} \varepsilon_{0}}{i_{0}}+\frac{C_{2} \varepsilon_{0}}{j_{0}} \quad \text { if } i \geq i_{0}, j \geq j_{0},
\end{aligned}
$$


where $C_{1}>0, C_{2}>0$ are constants.

Thus

$$
Q_{k i j}^{(m)} \subset\left\{\tau \in \Pi_{m}||-\langle k, \omega\rangle+l \mid<\frac{|l|+1}{A_{k}} \gamma_{m}+\frac{C_{1} \varepsilon_{0}}{i_{0}}+\frac{C_{2} \varepsilon_{0}}{j_{0}}\right\} \triangleq \widetilde{Q}_{k l},
$$

when $i \geq i_{0}, j \geq j_{0}$. By 10.6 , one has

$$
|l| \leq 4|\langle k, \omega\rangle| \leq C|k|
$$

Note that

$$
-\langle k, \omega\rangle+l=-\left\langle k, \omega_{0}\right\rangle \tau+l=\tau\left(-\left\langle k, \omega_{0}\right\rangle+\frac{l}{\tau}\right), \quad \tau \in[1,2] .
$$

Thus

$$
\widetilde{Q}_{k l} \subset\left\{\tau \in \Pi_{m}||-\left\langle k, \omega_{0}\right\rangle+\frac{l}{\tau} \mid<\frac{|l|+1}{A_{k}} \gamma_{m}+\frac{C_{1} \varepsilon_{0}}{i_{0}}+\frac{C_{2} \varepsilon_{0}}{j_{0}}\right\} \triangleq \widetilde{Q}_{k l}^{*} .
$$

Note

$$
\left|\frac{d}{d \tau}\left(-\left\langle k, \omega_{0}\right\rangle+\frac{l}{\tau}\right)\right|=\frac{|l|}{\tau^{2}} \geq \frac{1}{4}|l| .
$$

It follows that

$$
\operatorname{mes} \widetilde{Q}_{k l} \leq \operatorname{mes} \widetilde{Q}_{k l}^{*} \leq \frac{8}{|l|}\left(\frac{|l|+1}{A_{k}} \gamma_{m}+\frac{C_{1} \varepsilon_{0}}{i_{0}}+\frac{C_{2} \varepsilon_{0}}{j_{0}}\right)
$$

Take

$$
j_{0}=i_{0}=|k|^{n+1} \gamma_{m}^{-1 / 3}
$$

Then

$$
\begin{aligned}
\operatorname{mes} \bigcup_{1 \leq l \leq C|k|} \widetilde{Q}_{k l} & \leq \frac{C|k| \gamma_{m}}{A_{k}}+C \sum_{1 \leq|l| \leq C|k|} \frac{1}{|l|}\left(\frac{C_{1} \varepsilon_{0}}{i_{0}}+\frac{C_{2} \varepsilon_{0}}{j_{0}}\right) \\
& \leq \frac{C|k| \gamma_{m}}{A_{k}}+C \gamma_{m}^{1 / 3} \varepsilon_{0} \frac{\log |k|}{|k|^{n+1}} \\
& \leq C \gamma_{m}^{1 / 3} \varepsilon_{0} \frac{\log |k|}{|k|^{n+1}}
\end{aligned}
$$

Thus,

$$
\operatorname{mes} \bigcup_{\substack{i \geq i_{0} \\ j \geq j_{0} \\|i-j| \leq C|k|}} Q_{k i j}^{(m)} \leq C \gamma_{m}^{1 / 3} \varepsilon_{0} \frac{\log |k|}{|k|^{n+1}}
$$

Now assume

$$
i \leq i_{0} \quad \text { or } \quad j \leq j_{0} \quad \text { and } \quad|i-j| \leq C|k| \text {. }
$$


By 10.3 and 10.5, we have

$$
\begin{array}{r}
\left|\frac{d}{d \tau}\left(\frac{-\left\langle k, \omega_{0}\right\rangle \tau+\lambda_{i}^{(m)}-\lambda_{j}^{(m)}}{\tau}\right)\right| \geq \frac{\left|\lambda_{i}^{(m)}-\lambda_{j}^{(m)}\right|}{4} \geq \frac{|i-j|+1}{16}, \\
\operatorname{mes} \bigcup_{\substack{i \leq i_{0} \\
|i-j| \leq C|k|}} Q_{k i j}^{(m)} \leq \sum_{\substack{1 \leq i \leq i_{0} \\
|i-j| \leq C|k|}} \frac{2(|i-j|+1) \gamma_{m}}{A_{k}} \cdot \frac{16}{|i-j|+1} \\
\leq C i_{0} \frac{C|k| \gamma_{m}}{A_{k}} \leq C|k|^{n+2} \gamma_{m}^{2 / 3} \frac{1}{A_{k}} \leq \frac{C \gamma_{m}^{2 / 3}}{|k|^{n+1}}
\end{array}
$$

and

$$
\operatorname{mes} \bigcup_{\substack{j \leq j_{0} \\|i-j| \leq C|k|}} Q_{k i j}^{(m)} \leq \frac{C \gamma_{m}^{2 / 3}}{|k|^{n+1}}
$$

Combining 10.2, 10.7 10.8 and 10.9), we have

$$
\operatorname{mes} \bigcup_{|k| \leq K_{m}} \bigcup_{i=1}^{\infty} \bigcup_{j=1}^{\infty} Q_{k i j}^{(m)} \leq C \gamma_{m}^{1 / 3}
$$

Let

$$
\Pi_{m+1}^{+-}=\Pi_{m} \backslash \bigcup_{|k| \leq K_{m}} \bigcup_{i, j=1}^{\infty} Q_{k i j}^{(m)} .
$$

Then we have proved the following Lemma 10.1 .

\section{Lemma 10.1.}

$$
\operatorname{mes} \Pi_{m+1}^{+-} \geq \operatorname{mes} \Pi_{m}-C \gamma_{m}^{1 / 3}
$$

\section{Proofs of theorem and corollaries}

Proofs of Theorem 1.1 and Corollary 1.2, Let

$$
\Pi_{\infty}=\bigcap_{m=1}^{\infty} \Pi_{m} \text { and } \Psi_{\infty}=\lim _{m \rightarrow \infty} \Psi_{0} \circ \Psi_{1} \circ \cdots \circ \Psi_{m} .
$$

By (5.7) and (5.8), one has

$$
\Psi_{\infty}: \mathbb{T}^{n} \times \Pi_{\infty} \rightarrow \mathbb{T}^{n} \times \Pi_{\infty}, \quad\left\|\Psi_{\infty}-\mathrm{id}\right\|_{h_{N} \rightarrow h_{N}} \leq \varepsilon^{1 / 2}
$$

and, by (5.9),

$$
H_{\infty}=H \circ \Psi_{\infty}=\sum_{j=1}^{\infty} \lambda_{j}^{\infty} Z_{j} \bar{Z}_{j}
$$


where

$$
\lambda_{j}^{\infty}=\lim _{m \rightarrow \infty} \lambda_{j}^{(m)} .
$$

By 5.2 and (5.3), the limit $\lambda_{j}^{\infty}$ does exist and

$$
\lambda_{j}^{\infty}=j+O(\varepsilon / j):=\sqrt{j^{2}+\xi_{j}} .
$$

Introduce a transformation $\mathscr{G}: Z=\left(Z_{j} \in \mathbb{C}: j \geq 1\right) \mapsto v(t, x)$ by

$$
v(t, x)=\sum_{j=1}^{\infty} q_{j}(t) \sin j x, \quad Z_{j}=\frac{1}{\sqrt{2}}\left(q_{j}-\mathbf{i} p_{j}\right), \quad \bar{Z}_{j}=\frac{1}{\sqrt{2}}\left(q_{j}+\mathbf{i} p_{j}\right) .
$$

Let

$$
\Phi=\left(\mathscr{S} \mathscr{T} \mathscr{G} \Psi_{\infty} \mathscr{G}^{-1} \mathscr{T}^{-1} \mathscr{S}^{-1}\right)^{-1}
$$

Then $\Phi$ is a symplectic transformation and changes (1.3) subject to (1.4) into (1.5). Also, the transformation $\Phi$ changes the wave operator

$$
\mathscr{L}_{V}: \mathscr{L}_{V} u(t, x)=\left(\partial_{t}^{2}-\partial_{x}^{2}+\varepsilon V(\omega t, x)\right) u(t, x), \quad u(t,-\pi)=u(t, \pi)=0
$$

into

$$
\mathscr{L}_{M}: \mathscr{L}_{M} v(t, x)=\left(\partial_{t}^{2}-\partial_{x}^{2}+\varepsilon M_{\xi}\right) v(t, x), \quad v(t,-\pi)=v(t, \pi)=0,
$$

which possesses the property of pure point spectra and zero Lyapunov exponent.

This completes the proofs of Theorem 1.1 and Corollary 1.2 .

Proof of Corollary 1.3. By Theorem 1.1, we have that if $u=u(t, x)$ is a solution to (1.6), then

$$
v(t, x):=\left(\Phi\left(u, u_{t}\right)\right)(t, x)
$$

is the solution to 1.5 with

$$
\begin{aligned}
v(0, x) & =\left(\Phi\left(u, u_{t}\right)\right)(0, x)=\left(\Phi\left(u_{0}, \widetilde{u}_{0}\right)\right)(x), \\
v_{t}(0, x) & =\partial_{t}\left(\Phi\left(u, u_{t}\right)\right)(0, x)=\left(\Phi_{t}\left(u_{0}, \widetilde{u}_{0}\right)\right)(x) .
\end{aligned}
$$

Write

$$
v_{0}=v(0, x)=\sum_{k \in \mathbb{N}} C_{k} \sin k x, \quad \widetilde{v}_{0}=v_{t}(0, x)=\sum_{k \in \mathbb{N}} C_{k}^{\prime} \sin k x .
$$

By solving 1.5 directly, we have

$$
v(t, x)=\sum_{k=1}^{\infty}\left(C_{k}^{\prime}\left(\lambda_{k}+\varepsilon \xi_{k}\right)^{-1 / 2} \sin \left(\sqrt{\lambda_{k}+\varepsilon \xi_{k}} t\right)+C_{k} \cos \left(\sqrt{\lambda_{k}+\varepsilon \xi_{k}} t\right)\right) \sin k x .
$$


Note $k^{2}(1-c \varepsilon) \leq \lambda_{k}+\varepsilon \xi_{k} \leq k^{2}(1+c \varepsilon)$. It follows

$$
\begin{aligned}
\|v(t)\|_{\mathscr{H}^{N}}^{2} & =\sum_{k=1}^{\infty}\left(\left|C_{k}\right|^{2}+\left(\lambda_{k}+\varepsilon \xi_{k}\right)^{-1}\left|C_{k}^{\prime}\right|^{2}\right) k^{2 N} \\
& \leq \sum_{k=1}^{\infty}\left(\left|C_{k}\right|^{2}+(1+c \varepsilon) k^{-2}\left|C_{k}^{\prime}\right|^{2}\right) k^{2 N} \\
& =\left\|v_{0}\right\|_{\mathscr{H}^{N}}^{2}+(1+c \varepsilon)\left\|\widetilde{v}_{0}\right\|_{\mathscr{H}^{N-1}}^{2} .
\end{aligned}
$$

Noting $\mathscr{S}, \mathscr{T}, \mathscr{G}$ are isometric maps, using $\| \Psi_{\infty}-$ id $\|_{h_{N} \rightarrow h_{N}} \leq \varepsilon^{1 / 2}$, we have

$$
\begin{aligned}
\|u(t)\|_{\mathscr{H}^{N}}^{2} & \leq(1+c \sqrt{\varepsilon})\|v(t)\|_{\mathscr{H}^{N}}^{2} \leq(1+c \sqrt{\varepsilon})\left(\left\|v_{0}\right\|_{\mathscr{H}^{N}}^{2}+(1+c \varepsilon)\left\|\widetilde{v}_{0}\right\|_{\mathscr{H}^{N-1}}^{2}\right) \\
& \leq(1+c \sqrt{\varepsilon})\left(\left\|u_{0}\right\|_{\mathscr{H}^{N}}^{2}+\left\|\widetilde{u}_{0}\right\|_{\mathscr{H}^{N-1}}^{2}\right) .
\end{aligned}
$$

And

$$
\begin{aligned}
\|v(t)\|_{\mathscr{H}^{N}}^{2} & =\sum_{k=1}^{\infty}\left(\left|C_{k}\right|^{2}+\left(\lambda_{k}+\varepsilon \xi_{k}\right)^{-1}\left|C_{k}^{\prime}\right|^{2}\right) k^{2 N} \\
& \geq \sum_{k=1}^{\infty}\left(\left|C_{k}\right|^{2}+(1-c \varepsilon) k^{-2}\left|C_{k}^{\prime}\right|^{2}\right) k^{2 N} \\
& =\left\|v_{0}\right\|_{\mathscr{H}^{N}}^{2}+(1-c \varepsilon)\left\|\widetilde{v}_{0}\right\|_{\mathscr{H}^{N-1}}^{2},
\end{aligned}
$$

thus

$$
\|u(t)\|_{\mathscr{H}^{N}}^{2} \geq(1-c \sqrt{\varepsilon})\|v(t)\|_{\mathscr{H}^{N}}^{2} \geq(1-c \sqrt{\varepsilon})\left(\left\|u_{0}\right\|_{\mathscr{H}^{N}}^{2}+\left\|\widetilde{u}_{0}\right\|_{\mathscr{H}^{N-1}}^{2}\right),
$$

where $c$ is a positive constant which might be different in different places. Note

$$
v_{t}(t, x)=\sum_{k=1}^{\infty}\left(C_{k}^{\prime} \cos \left(\sqrt{\lambda_{k}+\varepsilon \xi_{k}} t\right)-C_{k} \sqrt{\lambda_{k}+\varepsilon \xi_{k}} \sin \left(\sqrt{\lambda_{k}+\varepsilon \xi_{k}} t\right)\right) \sin k x .
$$

Then

$$
\begin{aligned}
\left\|v_{t}(t)\right\|_{\mathscr{H}^{N-1}}^{2} & =\sum_{k=1}^{\infty}\left(\left(\sqrt{\lambda_{k}+\varepsilon \xi_{k}}\right)^{2}\left|C_{k}\right|^{2}+\left|C_{k}^{\prime}\right|^{2}\right) k^{2(N-1)} \\
& \leq \sum_{k=1}^{\infty}\left((1+c \varepsilon)\left|C_{k}\right|^{2} k^{2 N}+\left|C_{k}^{\prime}\right|^{2} k^{2(N-1)}\right) \\
& \leq(1+c \varepsilon)\left(\left\|v_{0}\right\|_{\mathscr{H}^{N}}^{2}+\left\|\widetilde{v}_{0}\right\|_{\mathscr{H}^{N-1}}^{2}\right) .
\end{aligned}
$$

Thus, by the preceding proof, we have

$$
(1-c \sqrt{\varepsilon})\left(\left\|u_{0}\right\|_{\mathscr{H}^{N}}^{2}+\left\|\widetilde{u}_{0}\right\|_{\mathscr{H}^{N-1}}^{2}\right) \leq\left\|u_{t}(t)\right\|_{\mathscr{H}^{N-1}}^{2} \leq(1+c \sqrt{\varepsilon})\left(\left\|u_{0}\right\|_{\mathscr{H}^{N}}^{2}+\left\|\widetilde{u}_{0}\right\|_{\mathscr{H}^{N-1}}^{2}\right) .
$$

This completes the proof of Corollary 1.3 . 


\section{Acknowledgments}

The work was supported in part by National Natural Science Foundation of China (Grants Nos. 11601277 and 11771253).

\section{References}

[1] P. Baldi, M. Berti and R. Montalto, KAM for autonomous quasi-linear perturbations of $K d V$, Ann. Inst. H. Poincaré Anal. Non Linéaire 33 (2016), no. 6, 1589-1638.

[2] D. Bambusi, Reducibility of 1-d Schrödinger equation with time quasiperiodic unbounded perturbations II, Comm. Math. Phys. 353 (2017), no. 1, 353-378.

[3] D. Bambusi and S. Graffi, Time quasi-periodic unbounded perturbations of Schrödinger operators and KAM methods, Comm. Math. Phys. 219 (2001), no. 2, $465-480$.

[4] D. Bambusi, B. Grébert, A. Maspero and D. Robert, Reducibility of the quantum harmonic oscillator in d-dimensions with polynomial time-dependent perturbation, Anal. PDE 11 (2018), no. 3, 775-799.

[5] M. Berti and P. Bolle, Sobolev quasi-periodic solutions of multidimensional wave equations with a multiplicative potential, Nonlinearity 25 (2012), no. 9, 2579-2613.

[6] N. N. Bogoljubov, Ju. A. Mitropoliskii and A. M. Samoĭlenko, Methods of Accelerated Convergence in Nonlinear Mechanics, Springer-Verlag, New York, 1976.

[7] J. Bourgain, Growth of Sobolev norms in linear Schrödinger equations with quasiperiodic potential, Comm. Math. Phys. 204 (1999), no. 1, 207-247.

[8] J. Bourgain and M. Goldstein, On nonperturbative localization with quasi-periodic potential, Ann. of Math. (2) 152 (2000), no. 3, 835-879.

[9] L. Chierchia and D. Qian, Moser's theorem for lower dimensional tori, J. Differential Equations 206 (2004), no. 1, 55-93.

[10] M. Combescure, The quantum stability problem for time-periodic perturbations of the harmonic oscillator, Ann. Inst. H. Poincaré Phys. Théor. 47 (1987), no. 1, 63-83.

[11] P. Duclos and P. Šťovíček, Floquet Hamiltonians with pure point spectrum, Comm. Math. Phys. 177 (1996), no. 2, 327-347. 
[12] P. Duclos, P. Šťovíček and M. Vittot, Perturbation of an eigen-value from a dense point spectrum: a general Floquet Hamiltonian, Ann. Inst. H. Poincaré Phys. Théor. 71 (1999), no. 3, 241-301.

[13] H. L. Eliasson and S. B. Kuksin, On reducibility of Schrödinger equations with quasiperiodic in time potentials, Comm. Math. Phys. 286 (2009), no. 1, 125-135.

[14] D. Fang, Z. Han and W.-M. Wang, Bounded Sobolev norms for Klein-Gordon equations under non-resonant perturbation, J. Math. Phys. 55 (2014), no. 12, 121503, 11 pp.

[15] R. Feola and M. Procesi, Quasi-periodic solutions for fully nonlinear forced reversible Schrödinger equations, J. Differential Equations 259 (2015), no. 7, 3389-3447.

[16] B. Grébert and E. Paturel, On reducibility of quantum harmonic oscillator on $\mathbb{R}^{d}$ with quasiperiodic in time potential, arXiv:1603.07455.

[17] B. Grébert and L. Thomann, KAM for the quantum harmonic oscillator, Comm. Math. Phys. 307 (2011), no. 2, 383-427.

[18] D. Jackson, The Theory of Approximation, Reprint of the 1930 original, American Mathematical Society Colloquium Publications 11, American Mathematical Society, Providence, RI, 1994.

[19] S. Klein, Anderson localization for the discrete one-dimensional quasi-periodic Schrödinger operator with potential defined by a Gevrey-class function, J. Funct. Anal. 218 (2005), no. 2, 255-292.

[20] S. B. Kuksin, Nearly integrable infinite-dimensional Hamiltonian systems, Lecture Notes in Mathematics 1556, Springer-Verlag, New York, 1993.

[21] Z. Liang and X. Wang, On reducibility of $1 d$ wave equation with quasiperiodic in time potentials, J. Dynam. Differential Equations 30 (2018), no. 3, 957-978.

[22] J. Liu and X. Yuan, Spectrum for quantum Duffing oscillator and small-divisor equation with large-variable coefficient, Comm. Pure Appl. Math. 63 (2010), no. 9, 11451172 .

[23] A. Maspero and D. Robert, On time dependent Schrödinger equations: global wellposedness and growth of Sobolev norms, J. Funct. Anal. 273 (2017), no. 2, 721-781.

[24] R. Montalto, A reducibility result for a class of linear wave equations on $\mathbb{T}^{d}$, Int. Math. Res. Not. IMRN 2019 (2019), no. 6, 1788-1862. 
[25] J. Pöschel, A KAM-theorem for some nonlinear partial differential equations, Ann. Scuola Norm. Sup. Pisa Cl. Sci. (4) 23 (1996), no. 1, 119-148.

[26] D. A. Salamon, The Kolmogorov-Arnold-Moser theorem, Math. Phys. Electron. J. 10 (2004), no. 3, 37 pp.

[27] D. Salamon and E. Zehnder, KAM theory in configuration space, Comment. Math. Helv. 64 (1989), no. 1, 84-132.

[28] Y. Sun, J. Li and B. Xie, Reducibility for wave equations of finitely smooth potential with periodic boundary conditions, Journal of Differential Equations 266 (2019), 27622804.

[29] W.-M. Wang, Pure point spectrum of the Floquet Hamiltonian for the quantum harmonic oscillator under time quasi-periodic perturbations, Comm. Math. Phys. 277 (2008), no. 2, 459-496.

[30] Z. Wang and Z. Liang, Reducibility of $1 D$ quantum harmonic oscillator perturbed by a quasiperiodic potential with logarithmic decay, Nonlinearity 30 (2017), no. 4, 1405-1448.

[31] X. Yuan and K. Zhang, A reduction theorem for time dependent Schrödinger operator with finite differentiable unbounded perturbation, J. Math. Phys. 54 (2013), no. 5, 052701, 23 pp.

[32] E. Zehnder, Generalized implicit function theorems with applications to some small divisor problems I, II, Comm. Pure Appl. Math. 28 (1975), 91-140; 29 (1976), no. 1, 49-111.

Jing Li

School of Mathematics, Shandong University, Jinan 250100, P. R. China

and

School of Mathematics and Statistics, Shandong University, Weihai 264209, P. R. China E-mail address: xlijing@sdu.edu.cn 